Cómo citar este artículo en Chicago: Chauta Velandia, Andrés Felipe. “Es Filolao el oponente del De prisca medicina?”. Escritos 29, no. 63 (2021): 264-286. doi: http://doi.org/10.18566/escr.v29n63.a05

Fecha de recepción: 02.03 .2021

Fecha de aceptación: 14.04.2021

\title{
¿Es Filolao el oponente del De prisca medicina?
}

\author{
Is Philolaus the opponent of De prisca medicina?
}

\author{
Andrés Felipe Chauta Velandia ${ }^{1}$
}

\section{RESUMEN}

La relación entre filosofía y medicina en la Antigüedad ha sido largamente tratada por comentaristas y estudiosos. El objetivo de este artículo fue determinar si es posible aseverar que el tratado hipocrático De prisca medicina (DM) sea una crítica dirigida a Filolao y, de ser posible, en qué términos podría estar planteada. Con esto en mente, me concentraré en la caracterización de la posición del oponente del autor de dicho tratado en DM $\$ 1.15$ -

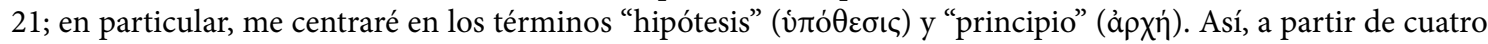
posibles interpretaciones propuestas del primero de estos términos, a saber: una proposición postulada pero que no es afirmada tajantemente, una proposición cuya veracidad es supuesta, una proposición que debe ser probada y un objeto puesto a discusión o una finalidad, esbozaré, asumiendo cada una de estas, las críticas que podrían estar en juego en DM y las contrastaré con la doctrina del filósofo pitagórico que puede ser reconstruida a partir de los fragmentos atribuidos a él como auténticos y algunos testimonios acerca de sus planteamientos médicos y epistemológicos. Finalmente, se enunció a modo de conclusión que el tratado hipocrático que es tematizado en él puede ser, en diversos modos, concebido como una crítica a Filolao. No obstante, es necesario resaltar que esta inquisición sobre este tema no tiene la intención de ser conclusiva; más bien, pretende otorgar elementos pertinentes a este debate.

Palabras clave: Filosofía; Epistemología; Medicina; Presocrática; Pitagorismo; Tratado; Filolao; Método; Hipótesis; Principio.

\section{ABSTRACT}

The relationship between philosophy and medicine in antiquity has been extensively discussed by commentators and scholars. The objective of this article was to determine if it is possible to assert that the Hippocratic treatise De prisca Medicina (DM) is a criticism directed at Philolaus and, if possible, in what terms it could be stated. With this in mind, the work concentrates on the characterization of the position of the opponent of the author of said

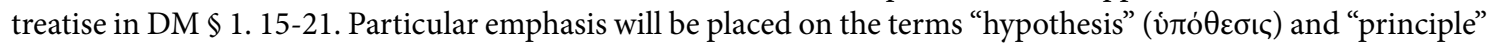

1 Filósofo por la Universidad Nacional de Colombia. Estudiante de posgrado en la Universidad Nacional de Colombia. Correo electrónico: afchautav@unal.edu.co 
(à $\rho \chi \eta \dot{)}$. Thus, from four possible proposed interpretations of the first of these terms, namely: a proposition postulated but not affirmed sharply, a proposition whose veracity is supposed, a proposition that must be proved, and an object put to discussion or a purpose, the criticisms that could be at stake in DM in each of these will be outlined and then contrasted with the doctrine of the Pythagorean philosopher that can be reconstructed from the fragments attributed to him as authentic, as well as some testimonies about his medical and epistemological approaches. Finally, it was stated by way of conclusion that the Hippocratic treatise that is thematized in it can be, in various ways, conceived as a criticism of Philolaus. However, it is necessary to emphasize that this inquisition on this subject is not intended to be conclusive; rather, it seeks to provide relevant elements to this debate.

Keywords: Philosophy; Epistemology; Medicine; Presocratic; Pythagoreanism; Treatise; Philolaus; Method; Hypothesis; Principle.

D eprisca medicina (DM) es un tratado hipocrático en el que el objetivo del autor no es la inquisición acerca de alguna enfermedad, sino que paralelamente a otros tratados del corpus hipocrático como De arte (DA) es la defensa de la medicina como técnica ( $\tau \dot{\varepsilon} \chi \nu \eta)$, de modo que encontramos una serie de argumentos y críticas encaminadas a demostrar su independencia, e incluso primacía, frente a la filosofía. Así, siguiendo a Schiefsky, se pueden distinguir dos momentos en la argumentación de DM: el primero, el cual se ubicaría en DM $\$ 3-8$, se centra en la demostración de que la medicina posee

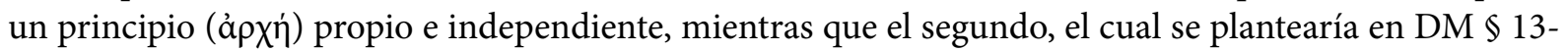
19, estaría dedicado a la refutación de las teorías fundamentadas en el planteamiento de las hipótesis

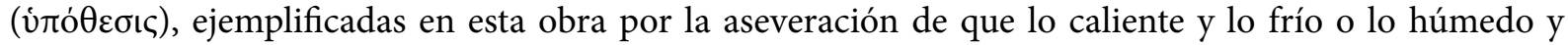
lo seco son causas primeras de las enfermedades. ${ }^{2}$ Por su parte, la primacía de la medicina frente a la filosofía es planteada en DM $\$ 2$ y $20 .^{3}$

Ahora bien, mucho se ha discutido sobre la identidad del oponente o los oponentes de DM, v. g., Diller ve en este tratado una crítica a los platónicos o a Platón mismo, en tanto estos harían uso del método hipotético que sería criticado en DM, ${ }^{4}$ mientras que Schiefsky no ve en DM un oponente concreto, sino más bien un movimiento médico. ${ }^{5}$ Además, Wanner ${ }^{6}$ junto con Wellmann ${ }^{7}$ plantean que la crítica estaría dirigida a alguien con influencia pitagórica. En esta misma línea, Lloyd, ${ }^{8}$ basándose en la aparición de los términos "frío y caliente" en el testimonio DK 44 A 27, y Huffman, ${ }^{9}$ en virtud de un uso del término

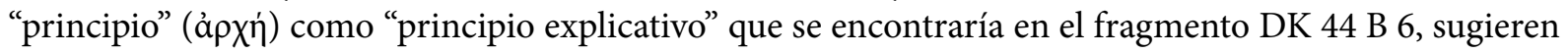
que el oponente que tendría en mente el autor de DM podría ser Filolao.

Dicho esto, mi propósito no es determinar quién o qué fue el objeto de crítica en $\mathrm{DM}$, más bien es determinar si Filolao pudo haber sido el oponente del autor de este tratado. Para esto, me centraré, principalmente, en la caracterización de las doctrinas que serán criticadas en DM que se encuentra en

2 Schiefsky, "Hipp. On An. Med., 25-36.

3 DM $\$ 2.8-9 ; \S 20.8-9$.

4 Diller, "Hipp. Med.Att. Phil., 385-409.

5 Schiefsky, "Hipp. On An. Med., 19-25.

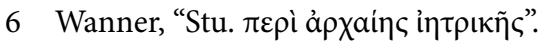

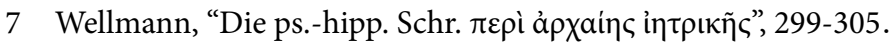

8 Lloyd, "Who Is Attacked in 'On Ancient Medicine'?", 108-126.

9 Huffman, "Philolaus of Croton: Pythagorean and Presocratic", 78-92. 
$\mathrm{DM} \S 1$. 15-21, en que se las presenta como las de aquellos que sostienen hipótesis (ن́tó $\theta \varepsilon \sigma ı \varsigma)$ en el ejercicio médico, de acuerdo con Schiefsky, tanto en su ámbito teórico como en el práctico, ${ }^{10}$ y en DM $\$ 20$, sección en la que se extiende explícitamente la crítica al ámbito de la filosofía. Por otra parte, cabe señalar que evitaré en lo posible hacer referencia a la mención de "lo frío y lo caliente" o "lo húmedo y lo seco" que se hace en DM $\$$ 1. 1-9 para justificar que Filolao sea el oponente del autor de DM. En ese sentido, también dejaré de lado el planteamiento del reduccionismo como característica de las posturas que serán criticadas en DM que señala Schiefsky, ${ }_{11}^{11}$ pues, tal como sostiene Huffman ${ }^{12}$ junto con Schiefsky ${ }^{13}$ y el mismo Lloyd ${ }^{14}$ reconoce, esto no es evidencia suficiente para concluir que Filolao es el

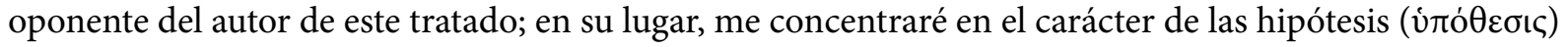
que estaría en juego en DM, cuyo uso es atribuido por el autor en DM $\$ 1,2,13$ y 20 a quienes plantean las teorías que serán criticadas. Así pues, con este objetivo, presentaré las posibles críticas al uso de hipótesis (ító $\theta \varepsilon \sigma \iota \varsigma)$, ya en la medicina, ya en la filosofía, que estarían planteadas en DM, asumiendo cada posible interpretación que, siguiendo a Lloyd, podría dársele a este término en el tratado que nos ocupa, a saber: a) una proposición postulada pero que no es afirmada tajantemente, b) una proposición cuya veracidad es supuesta, c) una proposición que debe ser probada y d) un objeto puesto a discusión o una finalidad, ${ }^{15} \mathrm{y}$ las contrastaré con el planteamiento de la doctrina de Filolao tanto en los fragmentos auténticos como en algunos testimonios, en atención a algunas de las interpretaciones que se le han dado

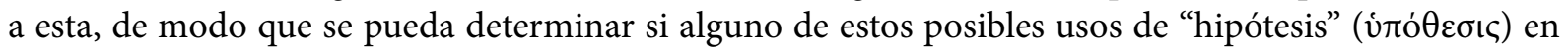
DM puede atribuirse a Filolao.

\section{I}

La primera de estas interpretaciones de "hipótesis" (ن́tó $\theta \varepsilon \sigma \iota \varsigma)$ es asociada por Lloyd ${ }^{16}$ con Jenófanes, quien en el fragmento DK 21 B 34 estaría, siguiendo a Schiefsky, enfatizando la idea de la imposibilidad de verificar la certeza de las proposiciones planteadas en las inquisiciones, que en todo caso serían posibles, sobre asuntos tales como las cosas del cielo o las que están debajo de la tierra, pues en este fragmento

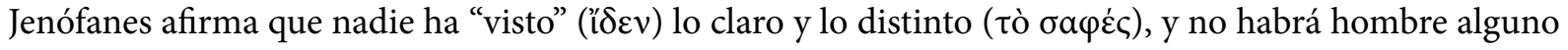
que lo vea respecto de los dioses y todas las cosas que él mismo, i. e., Jenófanes, dice sobre todos estos

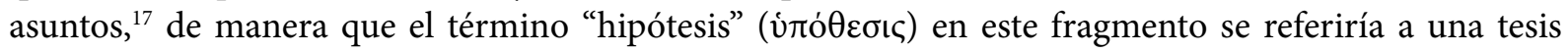
propuesta que, en tanto es incomprobable, no puede ser aseverada. ${ }^{18}$ Así, si se sostiene que este es el uso de hipótesis (író $\theta \varepsilon \sigma ı)$ que estaría en juego en DM, la crítica que se encuentra en este texto podría formularse en dos planos: uno epistemológico en el que se rechazaría la postulación de las hipótesis (vंđó $\theta \varepsilon \sigma ı \varsigma)$ en virtud de su incomprobabilidad, ya tomando la experiencia, ya la razón como criterio de

\footnotetext{
10 Schiefsky, "Hipp. On An. Med., 113.

11 Schiefsky, "Hipp. On An. Med., 57.

12 Schiefsky, "Hipp. On An. Med., 78-79.

13 Schiefsky, "Hipp. On An. Med.", 61.

14 Lloyd, "Who Is Attacked in 'On Ancient Medicine'?", 120.

15 Lloyd, "Who Is Attacked in 'On Ancient Medicine??", 110.

16 Lloyd, "Who Is Attacked in 'On Ancient Medicine??", 110.

17 DK 21 B 34.

18 Schiefsky, “Hipp. On An. Med.", 139-141.
} 


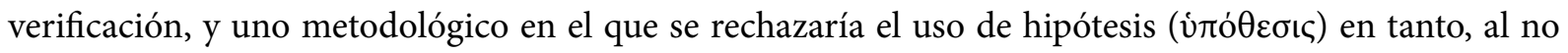
poder ser aseveradas, serían "superfluas" en la investigación.

No obstante, pese a que estas dos versiones de la crítica en DM según la interpretación del término "hipótesis" (ن́đó $\theta \varepsilon \sigma ı \varsigma)$ como una proposición que no es aseverada siguen la caracterización de los oponentes que serán objeto de crítica en el tratado que se encuentra en DM $\$ 1$. 15-21, pasaje en el que precisamente se alude al empleo de hipótesis (vंđó $\theta \varepsilon \sigma \iota \varsigma)$ en asuntos en los que no hay referencia alguna

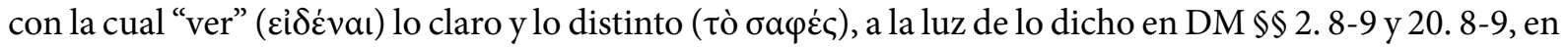
que se propone el método médico como único medio para la obtención certera de conocimiento acerca de cualquier asunto y, en ese sentido, se supone la comprobabilidad de las hipótesis (v́ró $\theta \varepsilon \sigma ı \varsigma)$ en todo ámbito de inquisición, solo la versión metodológica de la crítica sería plausible, pues, si se sostiene su versión epistemológica, se estaría planteando en DM la existencia de hipótesis incomprobables, incluso, mediante el método propio de la medicina, lo cual es contradictorio con lo dicho en estos pasajes. De este modo, se podría concluir, contrariamente a Schiefsky, quien propone que el autor de DM reconoce la validez del empleo de hipótesis en ciertos ámbitos de investigación, ${ }^{19}$ y junto con Lloyd, quien sostiene que el autor de DM le concede pertinencia al uso de hipótesis (v́ró $\theta \varepsilon \sigma \iota \varsigma)$ en la investigación acerca de los objetos del cielo y de la tierra solo irónicamente, ${ }^{20}$ que el autor de DM no asume la validez del uso de hipótesis (v́ró $\theta \varepsilon \sigma ı \varsigma)$ en ningún ámbito de la investigación y, si se sostiene esta interpretación, dado que el empleo del método médico tal como se lo plantea en DM $\$ \$ 2$ y 20 supone la comprobabilidad de cualquier proposición, el objeto de crítica en DM no puede ser el uso de proposiciones incomprobables; más bien, sería el uso de proposiciones que, en tanto no son aseveradas, serían superfluas en cualquier tipo de investigación.

Ahora bien, si aceptamos esta interpretación de "hipótesis" (vंđó $\theta \varepsilon \sigma \iota \varsigma)$, no se le podría atribuir su uso a Filolao en los fragmentos auténticos, pues, por un lado, aun cuando, dado que en el DK 44 B 6 sostiene que nada es cognoscible sobre la naturaleza ( $\varphi$ v́øı)) excepto que las cosas que existen y son conocidas

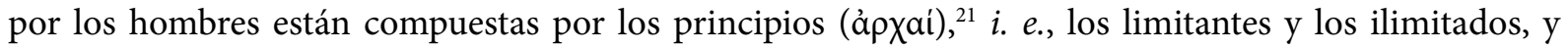
que, puesto que de otro modo no sería posible que el cosmos, en tanto orden, hubiera tenido lugar,

19 Schiefsky, "Hipp. On An. Med., 114.

20 Lloyd, "Who Is Attacked in 'On Ancient Medicine'?", 119.

21 Respecto del uso del término principio (ả $\rho \chi \eta \dot{)}$ en Filolao hay una discusión, puesto que se ha planteado que este podría usarse como principio explicativo, es decir, como una tesis planteada en un ámbito dialéctico como condición necesaria para que se dé algo -en Filolao, las cosas que son cognoscibles por el hombre, i. e., el cosmos como un todo y las cosas en él, serían incomprobables empíricamente y, en ese sentido, siguiendo a Huffman, concordarían con el carácter de las hipótesis (úđó $\theta \varepsilon \sigma \iota \varsigma)$ planteado en DM $\$ 1$ 15-21- y no como principio causal que, de hecho, dé lugar o constituya el orden cósmico y los objetos en este. Ahora bien, aunque se haga mención, de manera tangencial, de elementos que sean pertinentes a esta discusión, debo hacer explícito que no es mi intención tematizar este asunto, pues este requiere una inquisición más cuidadosa. No obstante, podría señalarse según los fragmentos del propio Filolao en lo que respecta a esta discusión que, aunque en DK 44 B 6 se encuentra un argumento que podría ser interpretado como puramente lógico y que concluiría tanto la existencia de los principios (ảpxaí) como la necesidad

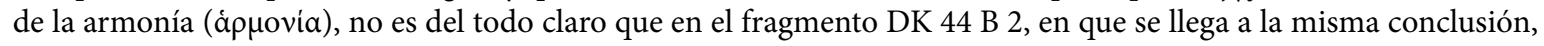
se dé un argumento de este mismo carácter, tal como, según Huffman, la interpretación estándar sostendría, pues, en este fragmento, hay una referencia a los hechos ( $\tau$ à $̌ ́ p \gamma a)$, la cual podría sugerir que la conclusión no se deriva de una necesidad lógica, sino de un cierto tipo de evidencia "experiencial". Para un tratamiento más cuidadoso de esta cuestión, véase Huffman "Philolaus of Croton: Pythagorean and Presocratic". 
es necesaria una cierta armonía ( $\dot{\alpha} \rho \mu o v i ́ a),{ }^{22}$ se puede sostener que este admite que hay cosas que son incognoscibles y, en ese sentido, se podría plantear que Filolao, al igual que Jenófanes en el fragmento DK 21 B 34, supondría que hay objetos sobre los cuales no es posible el conocimiento completo, i. e.,

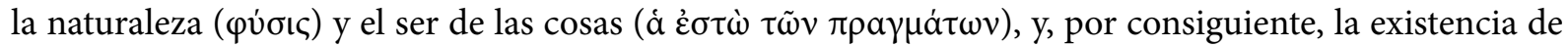
campos de inquisición física en los que cualquier proposición que se postule es incomprobable y, en tanto tal, superflua en la investigación, este se limita a presentar aquellas que son, según él, cognoscibles y, en otro orden de cosas, comprobables.

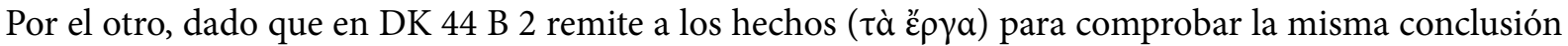
que se encuentra en DK 44 B 6, a saber: la existencia de los principios (ả $\rho \chi a i ́)$ y la necesidad de la armonía ( $\dot{\rho} \rho \operatorname{covi}^{\alpha}$ ), este supone la verificabilidad de las proposiciones que sostiene e, incluso, si seguimos a Huffman, ${ }^{23}$ se podría afirmar que Filolao, respondiendo a Jenófanes y con el objetivo de solucionar el problema epistemológico que supone el fragmento DK 21 B 34, el cual, además, estaría planteado, según Mourelatos ${ }^{24}$ y Kahn, ${ }^{25}$ en el poema de Parménides, postula el número como rasgo por medio del cual aquellos objetos que son oscuros están determinados y, en ese sentido, son cognoscibles.

En consecuencia, la crítica en DM planteada sobre la interpretación de "hipótesis" (v́ró $\theta \varepsilon \sigma ı \varsigma) ~ c o m o$ proposición no aseverada en virtud de su incomprobabilidad no podría estar dirigida a Filolao, dado que las proposiciones planteadas por Filolao no supondrían el carácter superfluo que sería atribuido en DM a las hipótesis, puesto que este no mantiene sus conclusiones como meras propuestas que no llega a afirmar, sino que aquellas que postula las plantea como necesariamente ciertas, ni sostiene ninguna del tipo de las que, a la luz del planteamiento de la incomprobabilidad de las doctrinas respecto de ciertos objetos incognoscibles propuesto por Schiefsky y Lloyd, estarían en juego en el fragmento DK 21 B 34 de Jenófanes, pues este, ya que remite a los hechos ( $\tau \grave{a}$ ع́p $\gamma \alpha$ ) para sostener sus tesis y las deduce argumentalmente, supone su comprobabilidad.

\section{II}

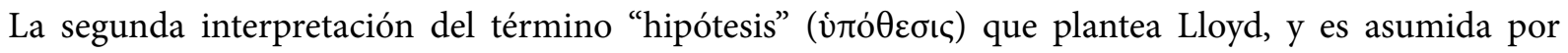
Longrigg, ${ }^{26}$ según la cual este refiere a una proposición cuya veracidad es supuesta, la postula Diller refiriéndose a una influencia platónica que estaría presente en $\mathrm{DM}$, de modo que sobre esta podría entenderse este término en el tratado como un supuesto, tal como, según este comentarista, este término se estaría planteando en el Fedón, el Menón o en el Parménides. ${ }^{27}$ Ahora bien, si seguimos esta interpretación, la crítica que encontramos en DM, dado que no estaría en juego la comprobabilidad o incomprobabilidad de la veracidad de la tesis supuesta como en la interpretación anterior, solo podría ser interpretada como una crítica metodológica al uso de supuestos en la inquisición, ya sea que esta se

22 DK 44 B 6.

23 Huffman, “The Role of Number in Philolaus' Philosophy”, 19-30.

24 Mourelatos, “The Route of Parmenides”, 47-73.

25 Kahn, “The art and thought of Heraclitus", 143-166.

26 Longrigg, "Greek Med. Her. Hell. Age", 58-59.

27 Diller, “Hipp. Med.Att. Phil.”, 385-409. 
limite al ámbito de la medicina, como, según Schiefsky, ${ }^{28}$ sucede al menos en DM $\$ 1$, ya sea que esta se extienda al ámbito de cualquier investigación como parecen sugerir DM $\$ \$ 2$ y 20 . En otro orden de ideas, el uso de supuestos, según Diller, sería rechazado por el autor de DM en tanto la medicina no tendría la necesidad de recurrir a estas hipótesis, las cuales serían propias de las investigaciones hechas en filosofía, como punto de partida en su investigación. ${ }^{29}$

Dicho esto, el planteamiento de Diller ha sido objeto de múltiples críticas, puesto que, por un lado, tal como señala Lloyd, la influencia platónica que supone y que lleva a pensar a este comentarista que el autor de $\mathrm{DM}$ en su caracterización de las hipótesis (író $\theta \varepsilon \sigma \iota \varsigma)$ tuvo en mente no solo un planteamiento platónico, sino a Platón mismo, es muy improbable; ${ }^{30}$ por otro, se puede argumentar, tal como señala Schiefsky, ${ }^{31}$ que tanto en el Fedón como en el Parménides, más que como tesis asumidas desde el principio, las hipótesis pueden ser entendidas, al menos en un contexto dialéctico, como proposiciones que serán objeto de evaluación, ${ }^{32}$ de manera que no se podría plantear esta interpretación de "hipótesis" (vंró $\left.\theta \varepsilon \sigma ı\right)$ ) en referencia a la presentación del método hipotético que aparece en estos diálogos.

Finalmente, cabe señalar que, aunque en el Menón, si seguimos a Robinson, ${ }^{33}$ se podría encontrar respecto del modo de proceder de los geómetras un uso de las hipótesis como las que plantea Diller, i.e., como supuestos sobre los cuales se fundamenta una inquisición, el cual, además, siguiendo a Burnyeat, ${ }^{34}$ podríamos encontrar en la República atribuido a los matemáticos, quienes usarían el término "hipótesis" (ن́đó $\theta \varepsilon \sigma \iota \varsigma)$ referido a definiciones de nociones fundamentales, tales como el número par, no es claro que este uso de las hipótesis pueda ser atribuido a Platón mismo, pues en la República Sócrates critica el proceder según hipótesis de los matemáticos y los geómetras, dado que en su proceder estas no solo serían tomadas como meros puntos de partida para las demostraciones, sino que también no darían cuenta de un conocimiento real acerca de lo que suponen, ${ }^{35}$ y si se está de acuerdo con lo que plantea Schiefsky, ${ }^{36}$ serían reconocidas por la dialéctica como meras fundamentaciones provisionales que deben ser reemplazadas por un principio no hipotético. En consecuencia, aunque el planteamiento de Diller no es claro en varios aspectos, tal como señala Lloyd, ${ }^{37}$ se podría rastrear un uso del término "hipótesis" (író $\theta \varepsilon \sigma \iota \varsigma)$ análogo al que plantea Diller, es decir, como supuestos fundacionales de inquisiciones, en los ámbitos de la geometría y las matemáticas, de modo que este proceder según supuestos pudo haber sido introducido en la medicina y ser el objeto de la crítica del autor de DM.

28 Schiefsky, “Hipp. On An. Med., 114.

29 Diller, “Hipp. Med.Att. Phil., 389-394.

30 Lloyd, "Who Is Attacked in 'On Ancient Medicine'?”, 122-123.

31 Schiefsky, “Hipp. On An. Med., 124.

32 Fed. 101d 3-5, Parm. 135e 8-136a 2.

33 Robinson, "Plato's Earlier Dialectic", 118-128.

34 Burnyeat, "Plato on Why Mathematics is Good for the Soul", 25-26.

35 Rep. 510 b $4-511 \mathrm{~d} 5$.

36 Schiefsky, "Hipp. On An. Med., 125.

37 Lloyd, “Who Is Attacked in 'On Ancient Medicine’?", 123-124. 
Por otra parte, Schiefsky ${ }^{38}$ atribuye a Diller una interpretación epistemológica según la cual en DM se estaría oponiendo la técnica ( $\tau \dot{\varepsilon} \chi \nu \eta)$ a la práctica ( $\dot{\varepsilon} \mu \tau \varepsilon \iota \rho i ́ \alpha)$, la cual refuta en tanto en DM, de hecho, no se daría esta oposición, sino que más bien se plantearía una oposición entre la técnica ( $\tau \dot{\varepsilon} \chi \vee \eta)$ y el

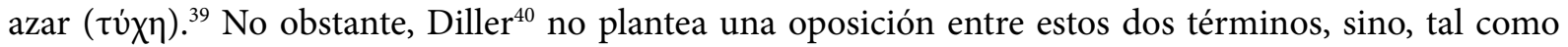
señala Lloyd, ${ }^{41}$ entre el método propio de la técnica ( $\left.\tau \dot{\varepsilon} \chi \vee \eta\right)$ y el método hipotético que sería, según él, propio de la filosofía, cuyo planteamiento ya fue expuesto. De modo que, dado que Diller no sostiene que se critique en DM la falta de desarrollo teórico de ciertas prácticas médicas, sino el planteamiento de hipótesis (író $\theta \varepsilon \sigma \iota \varsigma)$, cuya veracidad en tanto supuesta no es objeto de examen ni empírico ni racional en el desarrollo de la medicina, la interpretación de la crítica propuesta por Diller no sería, en principio, la objeción metodológica o epistemológica a la práctica $(\dot{\varepsilon} \mu \pi \varepsilon \iota \rho i \alpha)$, que, según Schiefsky, se estaría planteando a partir de DM $\$ 9$ según distinción entre la práctica $(\varepsilon \dot{\varepsilon} \mu \varepsilon \iota \rho i ́ a)$, la técnica ( $\tau \dot{\varepsilon} \chi \vee \eta)$ y la ciencia $(\dot{\varepsilon} \pi \iota \tau \tau \dot{\mu} \mu)$ en el Filebo. ${ }^{42}$

Ahora bien, aunque Diller, ${ }^{43}$ basado en el Gorgias y el Filebo, en que, como ya se ha resaltado, se plantea la distinción entre las técnicas ( $\tau \dot{\varepsilon} \chi \vee \propto \iota)$ y las prácticas ( $\left.\dot{\varepsilon} \mu \pi \varepsilon ı \rho^{\prime} a l\right)$ en virtud de que las primeras hacen

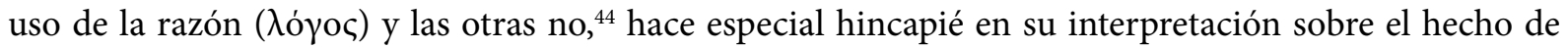
que el planteamiento de las hipótesis (író $\theta \varepsilon \sigma \iota \varsigma)$, que, a la luz de estos diálogos, sería atribuido por Platón a la medicina en tanto técnica ( $\tau \dot{\varepsilon} \chi \nu \eta)$, contrariamente a como se haría en la medicina, tal como la entiende el autor de DM, i. e., una técnica ( $\tau \dot{\varepsilon} \chi \nu \eta)$ cuyo criterio ( $\mu \varepsilon \dot{\tau} \tau o v)$ de verificación, en principio,

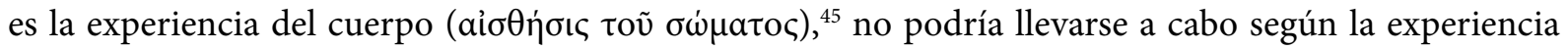

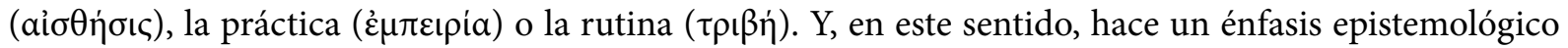
en su interpretación, énfasis que sería pertinente solo dentro de una crítica metodológica, es decir, solo se hace énfasis en la experiencia en tanto el método propio de la medicina para el autor de DM sería “empírico” y no hipotético.

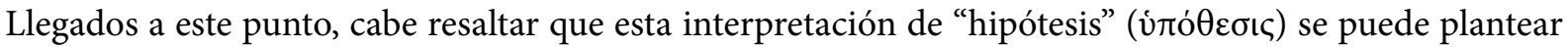
de otro modo, a saber: poniéndolo en relación con el término "principio" (ả $\rho \chi \eta ́)$, esto según De carnibus (DC), otro tratado hipocrático en que aparecen ambos términos y se nos dice:

38 Schiefsky, "Hipp. On An. Med., 47.

39 Esta es una contraposición que se plantea también en De arte, tratado en el cual se argumenta la existencia de la medicina como técnica ( $\tau \dot{\varepsilon} \chi \nu \eta)$ opuesta a la curación resultado del azar ( $\tau \dot{\chi} \chi \eta)$ sobre la base de que la intervención médica no es irrelevante frente al acontecer por azar ( $\tau \dot{x} \chi \eta)$ o casualidad; dicho de otro modo, la curación no puede ser atribuida al azar ( $\tau \dot{\chi} \chi \eta)$, puesto que, por un lado, aunque este desempeñe un papel en este proceso, el paciente que es curado lo es por un tratamiento médico o, en caso de que no recurra a un médico, a un tratamiento tal como lo recetaría un médico; por otro, es posible distinguir un buen tratamiento de uno malo, lo cual no sería posible en el caso de que la curación fuera resultado del puro azar ( $\tau u ́ x \eta)$; véase DA $₫ 4$-5.

40 Diller, “Hipp. Med.Att. Phil., 385-409.

41 Lloyd, "Who Is Attacked in 'On Ancient Medicine’?", 118-119.

42 Fil. 55e 1-56c 7.

43 Diller, “Hipp. Med.Att. Phil.”, 399-401.

44 Gorg. 463a 6-b 4 y 500e 3-501a 3; Fil. 55e 1-56c 7.

45 DM $\$ 9.11-13$. 


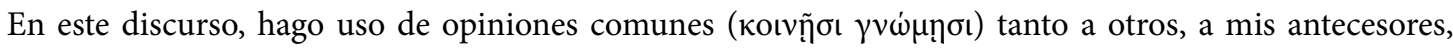

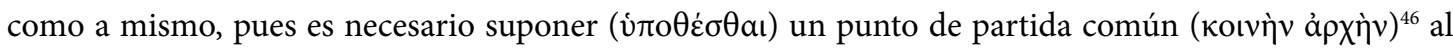
componer este tratado del arte médico. Sobre las cosas en el cielo no tengo necesidad de hablar, excepto en tanto, como voy a mostrar, respecto del ser humano y de otras cosas vivientes cómo se originaron y llegaron a ser, qué es el alma, qué es la salud y la enfermedad, qué es en el hombre lo bueno y lo malo y por qué razón $<$ este $>{ }^{47}$ muere. ${ }^{48}$

A la luz de esta pasaje de DC, tal como señala De la Villa Polo,${ }^{49}$ es evidente el paralelismo entre la manera de proceder en la medicina que plantea el autor de DC y la caracterización de los oponentes de DM, pues, en ambos tratados, se nos presenta un método médico, que, por un lado, está basado en suposiciones

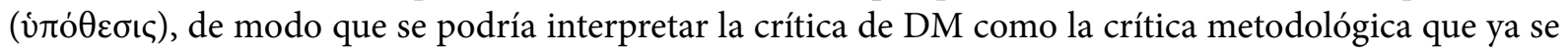
ha planteado refiriéndose a Diller, a saber: la crítica al planteamiento de supuestos en la medicina; por

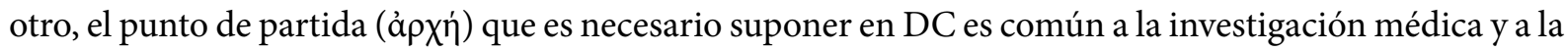
que se ocupa de los asuntos del cielo, y en ese sentido, corresponde con la caracterización de las hipótesis

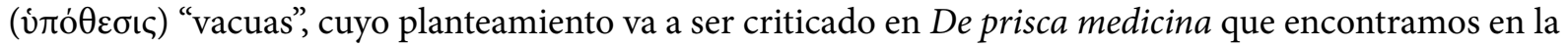
parte final de DM $\$ 1 .^{50}$

Dicho esto, se podría plantear, por un lado, junto con Schiefsky, ${ }^{51}$ que la crítica que hace el autor en DM está dirigida a la influencia filosófica que pondría en tela de juicio la independencia de la medicina como técnica ( $\tau \dot{\varepsilon} \chi \vee a ı)$ y se manifestaría en esta como el planteamiento de una etiología, una patología, un diagnóstico y un tratamiento de las enfermedades basado en tesis embriológicas o "antropogónicas", $i$. e., el tipo de planteamientos que serían propios de la filosofía presocrática que busca el principio último (åpx门́) del cosmos y del hombre en tanto parte de este; por el otro, junto con Lloyd, ${ }^{52}$ y de acuerdo con lo que se ha planteado más arriba en referencia a la interpretación del Menón y la República que sostiene Robinson y Burnyeat, que esta crítica está dirigida a la influencia de planteamientos de la geometría o de las matemáticas en la medicina. Ahora bien, cabe remarcar que estas interpretaciones de la crítica concordarían con el desarrollo de las críticas y el planteamiento de los principios médicos que se encuentran en DM, puesto que en la primera parte de este tratado se argumenta que la medicina

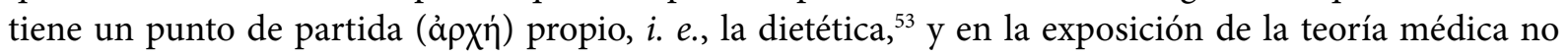
hace referencia ni a la teoría humoral ni a definiciones matemáticas o a principios "embriológicos" y "antropogónicos", sino que refiere a términos propios de la dietética, tales como lo dulce, lo salado, lo amargo, etc. ${ }^{54}$

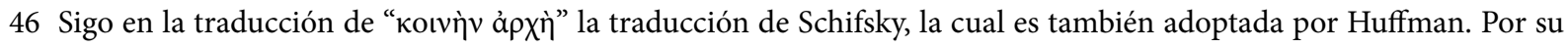
parte, De la Villa Polo traduce como "unos principios comunes".

47 Paréntesis angulares agregados por mí.

48 DC \$1. 1-8. Traducción mía.

49 De la Villa Polo, "Sobre las carnes", 143.

$50 \mathrm{DM} ₫ 1.15-21$.

51 Schiefsky, "Hipp. On An. Med., 19-25; Schiefsky, "On Anc. Med. on the nat. of human beings", 69-85.

52 Lloyd, "Who Is Attacked in 'On Ancient Medicine?", 122-123.

$53 \mathrm{DM} ₫ 2-4$.

$54 \mathrm{DM} \$ 1420-27$. 


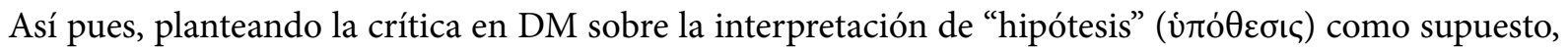
el oponente de DM no sería necesariamente alguien con filiación platónica o Platón mismo en tanto proceden de acuerdo con el método hipotético, que, como lo plantea Diller, podría encontrarse en algunas de las obras de este último, sino que también podría ser tanto un médico como el autor de DC, cuyo planteamiento médico supone un punto de partida (ả $\rho$ ท́) común con la investigación acerca de los objetos del cielo y de debajo de la tierra, sea esta filosófica, matemática o geométrica, como un filósofo físico, un matemático o un geómetra, cuya teoría a este respecto habría permeado en su doctrina médica.

Hecha esta presentación de las críticas que se podrían encontrar en DM, a saber: una al método hipotético filosófico o "matemático" y otra a la suposición de un punto de partida para la medicina que estaría basado, o bien en nociones matemáticas, o bien en la geometría misma o en las tesis embriológicas, antropogónicas o cosmogónicas que serían propias de la filosofía, es necesario señalar que Filolao como filósof ${ }^{55}$ no podría ser el oponente que el autor de DM tendría en mente al escribir el tratado, pues, aún asumiendo que en el fragmento DK 44 B 6, tal como propone Huffman, ${ }^{56}$ se entienda el término

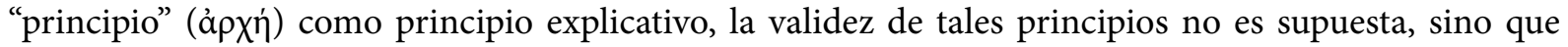
se concluye, ya por un medio lógico, como sería el caso en DK 44 B 6 y en DK 44 B 2, si se sigue la interpretación estándar que presenta $\mathrm{Huffman}^{57}$ en su comentario a este fragmento, ya si se concluyen por medios ajenos al lógico, tal como se podría llegar a sostener por la referencia a los hechos ( $\tau a ̀$ ép $p \alpha$ ) en DK 44 B 2.

No obstante, se podría sostener según DK 44 A 27 que Filolao como médico sí podría ser el oponente que es criticado en DM en tanto este podría estar asumiendo tesis filosóficas, las cuales, a la luz de la interpretación propuesta en esta sección de la crítica en DM, podrían clasificarse como embriológicas o antropogónicas, como punto de partida para una inquisición médica, ya que, si seguimos el planteamiento de Huffman, ${ }^{58}$ se puede sostener que, en tanto, por un lado, en ambas doctrinas se hace referencia a un elemento externo que determina u organiza, ya el cosmos, ya el cuerpo del embrión; por otro, aunque en los fragmentos auténticos de Filolao no hay mención explícita ni al fuego ni a lo caliente como principio

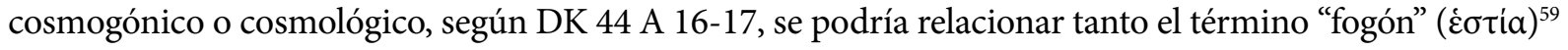
que aparece en DK 44 B 7 con el fuego, relación que concordaría con el reporte que hace Aristóteles en $D e$ caelo sobre la teoría cosmológica de los pitagóricos, quienes, según este pasaje, sostienen que en el centro hay fuego, ${ }^{60}$ como el fuego con lo caliente en DK 44 A 27, la teoría cosmogónica y cosmológica que se encontraría en el fragmento DK 44 B 17, en que se encontraría la tesis de que las cosas en el cosmos serían

55 Se podría sostener que Filolao afirma sin demostración, al menos, la necesidad del número para el conocimiento que es planteada en DK 44 B 4 . Sin embargo, dado el estado fragmentario de la obra de este autor, tal como señala Lloyd y Huffaman, es imposible asegurar que Filolao haya, de entrada, tomado, en modo análogo a los geómetras, a los cuales, según Robinson y Burnyeat, Platón en el Menón y en la República atribuiría un método que parte de la suposición de nociones tales como el número par e impar como supuesto necesario al número.

56 Huffman, "Philolaus of Croton: Pythagorean and Presocratic", 78-92.

57 Huffman, "Philolaus of Croton: Pythagorean and Presocratic", 102-107.

58 Huffman, "Philolaus of Croton: Pythagorean and Presocratic", 41-47.

59 Sigo en la traducción de este término en DK 44 B 7 la traducción alemana de Diels-Kranz, quienes lo traducen como "Herd", vocablo que se puede traducir al español como "fogón" o "punto de partida". Por su parte, Huffman lo traduce como "hearth" y Eggers lo traduce como "hogar".

60 De caelo I 293a 20-23. 
determinadas desde el exterior, y la teoría médica que encontramos en el DK $44 \mathrm{~A} 27$, en que se hace referencia a la respiración como medio por el cual lo caliente y lo frío se "armonizan" en los recién nacidos, serían análogas. Finalmente, se puede señalar, tal como lo hace Huffman, ${ }^{61}$ que, dado que en este testimonio se sostiene la necesidad de un "balance" entre lo frío y lo caliente en el cuerpo, se podría plantear que la teoría médica de Filolao supone la noción de armonía (åpuovía) que está presente en los fragmentos DK 44 B 1,2,6 y 7. De modo que, aun si no se acepta la analogía propuesta por Huffman entre DK 44 B 17 y DK 44 A 27, la teoría médica que se encuentra en este testimonio estaría planteada según tesis filosóficas.

Además, se podría aseverar que Filolao como médico puede ser el oponente del autor de DM, si se interpreta su crítica como una dirigida a la suposición de un punto de partida común a la medicina y a la filosofía, puesto que se puede plantear, junto con Lloyd, ${ }^{62}$ quien lo hace a la luz del fragmento DK $44 \mathrm{~A} 7 \mathrm{a}$, en que, de acuerdo con Huffman, ${ }^{63}$ se sostiene que la geometría es el principio (ả $\left.\rho x \eta ́\right)$ de las ciencias matemáticas, según este testimonio y el fragmento DK 44 B 4, en el cual, siguiendo a Huffman, ${ }^{64}$ se estaría planteando el número en tanto medio de expresión del orden y proporciones de todas las cosas en el cosmos y del cosmos mismo como requisito y medio para el conocimiento certero de todo lo que puede ser conocido, que Filolao sostiene, por un lado, que la geometría es una base necesaria para el conocimiento de cualquier asunto, y por otro, que el número es principio del conocimiento. Y, en ese sentido, siguiendo a Huffman, este al formular tanto su teoría médica como su doctrina filosófica tendría

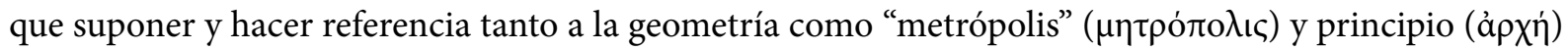
de la investigación en cualquier ámbito, ${ }^{65} i$. e., de acuerdo con Huffman, como su punto de origen y continuo soporte, y tal como señala Huffman, ${ }^{66}$ como al número como base epistemológica.

En consecuencia, el oponente de DM, si se sostiene que en este tratado se estaría criticando el planteamiento de la medicina sobre el supuesto de un punto de partida (ả $\rho \chi \eta \dot{)}$ común a la filosofía y a la medicina, podría ser Filolao como médico, pues, incluso si no se siguen las analogías planteadas por Huffman

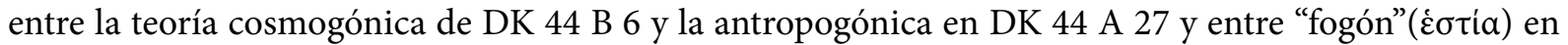
DK 44 B 7, el fuego y lo caliente, este estaría suponiendo la necesidad de una armonía (ápuovía) tanto en su doctrina filosófica que se encuentra en los fragmentos auténticos como en su teoría médica en el testimonio citado. Y se podría sostener, según DK 44 A 7a y a DK 44 B 4, que este supone que la geometría y las nociones matemáticas son puntos de partida para cualquier tipo de conocimiento certero, incluidas

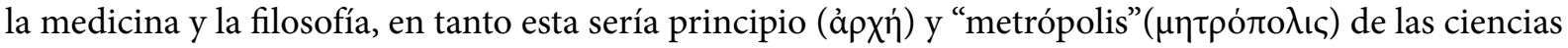
matemáticas y solo a través del número sería posible conocer algo. ${ }^{67}$ Por el contrario, si se toma la crítica

61 Huffman, "Philolaus of Croton: Pythagorean and Presocratic", 87-88.

62 Lloyd, "Who Is Attacked in 'On Ancient Medicine'?”, 122-123.

63 Huffman, "Philolaus of Croton: Pythagorean and Presocratic", 193-199.

64 Huffman, "Philolaus of Croton: Pythagorean and Presocratic", 19-30.

65 En el fragmento, aparece propiamente el termino “ $\mu \alpha \theta \eta \dot{\mu \alpha \tau \alpha ”, ~ e l ~ c u a l, ~ e n ~ e l ~ c o n t e x t o ~ d e l ~ f r a g m e n t o, ~ e s ~ a m b i g u o . ~}$ No obstante, siguiendo el comentario de Huffman, quien remite al fragmento DK 47 B 1 de Arquitas de Tarento, se podría conjeturar que con este término Filolao se refiere a la astronomía, la música, la geometría, la aritmética y, posiblemente, a cualquier ámbito de conocimiento con una descripción de su objeto investigación, de modo que podría incluir ciencias tales como la medicina.

66 Huffman, "Philolaus of Croton: Pythagorean and Presocratic", 196; Huffman, "The Role of Number in Philolaus' Philosophy", 19-30.

67 Véase la nota al pie de página 63. 
de DM como crítica al método hipotético propio de la filosofía, esta no podría estar dirigida a Filolao como filósofo, ya que en los fragmentos atribuidos a este autor no se supone la veracidad de la tesis que afirma la necesidad de los principios (ả $\rho \chi \alpha^{\prime}$ ); esta, en efecto, es justificada, ya por medios lógicos, ya por medios "experienciales".

\section{III}

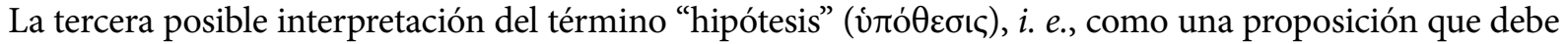
ser probada, la presenta Lloyd acudiendo al sentido que tiene este término en De flatibus (DF), tratado en el cual, tal como señala Schiefsky, se presenta, argumenta y concluye la validez de una hipótesis (író $\theta \varepsilon \sigma ı){ }^{68}$ Ahora bien, esta interpretación ha sido considerada por varios comentaristas, incluido Lloyd, ${ }^{69}$ como inapropiada para DM, en tanto en este se estaría entendiendo "hipótesis" (író $\theta \varepsilon \sigma \iota \varsigma)$ como un supuesto, mientras que en DF, tal como se ha señalado, no se daría por sentada la validez de tal proposición.

Dicho esto, cabe resaltar que, dado que, por un lado, en DM $\$ 2.8-9$ y 20. 8-9 se presenta el camino de la medicina como el único medio para obtener conocimiento, y en ese sentido, tal como sostiene Dunn, ${ }^{70}$ en tanto hay cierta esperanza por parte del autor en que por medio del método "empírico" eventualmente se alcanzará conocimiento, incluso, acerca de los asuntos propios de la filosofía, a saber: los asuntos del cielo y de debajo de la tierra, en que, como ya se ha señalado, no hay, en principio, nada claro y distinto, la idea de un "progreso intelectual" en cualquier ámbito de investigación estaría presente en DM. Por otro lado, puesto que, de hecho, en DM las critica y demuestra, de acuerdo con el método "empírico", que, según Diller ${ }^{71}$ y Schiefsky, ${ }^{72}$ sería propio de la medicina, que son superfluas en el ejercicio médico en su ámbito tanto teórico como práctico, ya que estas no darían cuenta del origen de las enfermedades y no son útiles ni en el diagnóstico ni en el tratamiento, ${ }^{73}$ el planteamiento de las hipótesis (író $\left.\theta \varepsilon \sigma ı\right)$ en $\mathrm{DM}$, tal como ya se ha señalado en $\$ \mathrm{I}$, supone su comprobabilidad, incluso, en las inquisiciones en que no hay, en principio, criterio de verificación. En consecuencia, en referencia a lo dicho en DM, no habría,

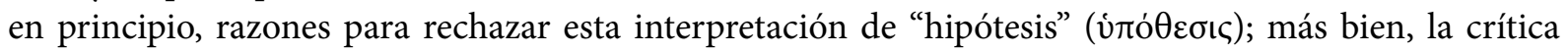
planteada en DM $\$ 2,13-19$ y 20 supondría la comprobabilidad de todas proposiciones.

En otro orden de cosas, se podría interpretar la crítica en DM, por una parte, siguiendo a Schiefsky y a DM

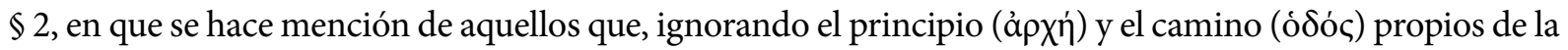
medicina, investigan con otros esquemas y métodos, ${ }^{74}$ como una crítica que se dirigiría al planteamiento del ejercicio médico, que se fundamenta según hipótesis (ító $\theta \varepsilon \sigma \iota \varsigma)$ innecesarias y ajenas a la medicina sujetas a verificación, es decir, no son supuestos cuya veracidad se da por sentada, y en tanto tales son criticadas y

68 DF $\$ 151251$.

69 Lloyd, "Who Is Attacked in 'On Ancient Medicine'?”, 110.

70 Dunn, “On anc. med. and its intellectual context.", 49-67.

71 Diller, "Hipp. Med.Att. Phil., 399-401.

72 Schiefsky, “Hipp. On An. Med., 345-359.

$73 \mathrm{DM} \$ 13-19$.

$74 \mathrm{DM} \$ 2.4-7$. 
desechadas en $\mathrm{DM} ;{ }^{75}$ por otra, como una crítica al método hipotético, en tanto, según el autor de DM, solo a través del método "empírico" sería posible llegar a un conocimiento certero acerca de algún asunto, ${ }^{76}$ es decir, desde el punto de vista de DM $\$ 1$, solo en este método habría un criterio ( $\mu \varepsilon \dot{\tau} \rho \circ v$ ) para distinguir la

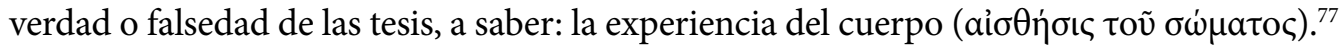

Dicho esto, la crítica de DM no podría estar dirigida al uso de hipótesis (ن́tó $\theta \varepsilon \sigma ı \varsigma)$, como la que se encuentra en DF, ya que en este, en principio, se justifica el planteamiento del aire como principio (ả $\rho \chi \eta$ ) de todas las enfermedades recurriendo al método "empírico". En efecto, el autor de DF recurre a observaciones para sostener, por ejemplo, que el aire es la causa de la vida, pues no se puede vivir sin respirar; ${ }^{78}$ más bien, la crítica a DF que el autor de DM podría formular versaría alrededor de veracidad de la hipótesis de que el aire sea principio de todas las enfermedades, doctrina que seguramente sería rechazada no según el modo de su planteamiento, como supondría quien aceptara que en DM se criticara el planteamiento

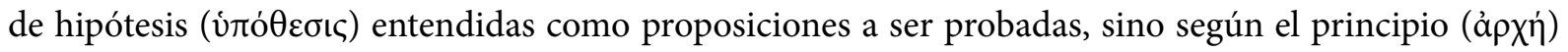
que es propuesto como causa de las enfermedades, demostrando en el proceso, análogamente a como lo hace en DM $\$ 13-19$, que el aire no da cuenta del origen de todas las enfermedades y que la referencia a principios cosmogónicos no es necesaria, en tanto la medicina dispondría de un trasfondo teórico, un

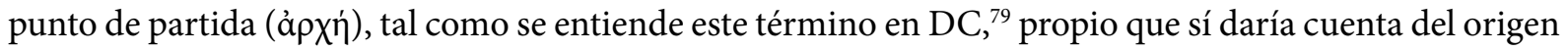
de las enfermedades y sobre el cual sería posible diagnosticarlas y tratarlas de manera pertinente.

Llegados a este punto, es necesario aclarar qué habría que entender por "método empírico" en DM. Pues bien, si se lo entiende como un método que recurre exclusivamente a la percepción (aiơnjoıs), como sería caracterizado el "método" de la práctica ( $\left.\varepsilon \mu \pi \varepsilon \iota i^{\prime} \alpha\right)$ por Platón, ${ }^{80}$ el planteamiento del procedimiento analógico que, tal como se lo presenta en $\mathrm{DM} \$ 22$, es apropiado tanto para la patología y el diagnóstico como para el tratamiento de las enfermedades causadas por las estructuras internas del cuerpo, cuyo funcionamiento no es visible, i. e., no es perceptible, sería problemático, ya que la veracidad del diagnóstico y la de la patología basada en esta manera de proceder serían incomprobables por medio de la percepción (aỉ $\theta \dot{j} \sigma ı \varsigma$ ). De otro modo, en tanto serían perceptibles, no habría necesidad de este método para el diagnóstico, el tratamiento y la patología de tales enfermedades, dado que, tal como en el caso de las enfermedades visibles, las causas de estas serían evidentes. ${ }^{81}$ Además, puesto que, tal

$75 \mathrm{DM} \S 13-19$.

$76 \mathrm{DM} \$ 2.8-9 ; \$ 20.8-9$.

$77 \mathrm{DM} \$ 9.11-13$.

$78 \mathrm{DF} \$ 4$.

79 DC $\$ 1.1-8$.

80 Gorg. 463a 6-b 4 y 500e 3-501a 3; Fil. 55e 1-56c 7.

81 Galeno en su obra De sectis ad eos qui introducuntur (DS) recoge, en su recuento del debate entre la secta médica empírica y la racionalista, el cual debe entenderse, según Frede, en el contexto de la ruptura entre la medicina tradicional y el desarrollo de una "nueva" técnica médica en el siglo V a. C., en el que, según este comentarista, estaría inmerso el autor de DM, una crítica similar por parte de los empiristas, a quienes Galeno caracteriza como aquellos que sostienen como fuente exclusiva para el conocimiento médico a la observación empírica, al procedimiento por analogías que sostienen los racionalistas entre cosas que son manifiestas y otras que no lo son, puesto que, en tanto las cosas deben ser conocidas por sí mismas, la analogía no estaría en "posición" de develar aquello que debería poder develar y, en ese sentido, o bien no proveería conocimiento cierto de aquello que no es manifiesto, o bien el conocimiento al que se llegaría sería superfluo. Véase Frede, "Galen: Three treatises On the Nature of Science”, XX-XXI. 
como señala Schiefsky, la medicina se restringiría a un campo "irracional” en tanto el ámbito médico tendría que abstenerse de cualquier modo de teorización, es decir, no podría remitir a ningún tipo de esquema teórico o metodológico, por un lado, la caracterización de los oponentes del autor en DM $\$ 2$. 4-7 como los que ignoran el método y los esquemas propios de la medicina no tendría sentido, ya que, en todo caso, esta no podría remitirse a estos en su ejercicio; por otro, ya que tales postulaciones implican una construcción teórica, que, según el autor de DM, es propia de la medicina, tanto la postulación de elementos tales como lo dulce, lo amargo, lo salado, etc., como la de los principios activos $(\delta v v a ́ \mu ı \varsigma)^{82} \mathrm{y}$ las estructuras internas del cuerpo como causas de las enfermedades ${ }^{83}$ serían problemáticas.

No obstante, en DM no parece ser esta la noción de "método empírico" que está en juego, pues, si consideramos lo dicho en DM $\$ 2$, en que se demuestra que no es necesario el uso de hipótesis (v́ró $\theta \varepsilon \sigma ı)$ ) en referencia a un modo de diagnosticar en el que tanto el paciente como el médico intervienen, el uno reportando sus síntomas y el otro explicando y descubriendo el padecimiento, sus causas y cómo ha de cesar, ${ }^{84}$ la misma idea que se encuentra en otros pasajes del texto, e. $g$., mientras que en DM $\$ 9$ se hace

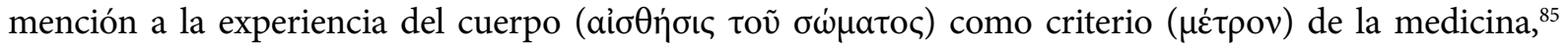

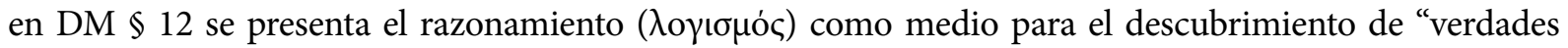
médicas", ${ }^{86}$ es posible plantear junto con Schiefsky ${ }^{87}$ en DM un empirismo que no apela meramente a la percepción del médico, sino también a su "experiencia técnica", la cual no puede reducirse ni a su ámbito puramente teórico, en el cual se incluirían las teorías y los descubrimientos médicos, ni a su ámbito puramente empírico, puesto que el conocimiento teórico solo podría llegar a ser verdaderamente útil para el médico cuando está acompañado de la práctica, i. e., el tratamiento y diagnóstico de pacientes, y esta, según DM $\$ 2$, requeriría tanto conocimiento teórico como la información que provee el paciente a partir

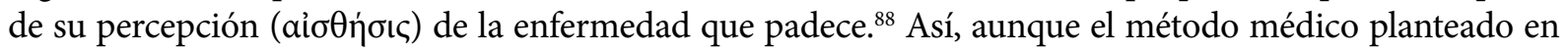

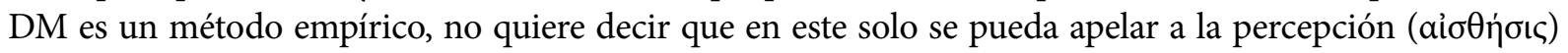
para comprobar si un diagnóstico es correcto o no; más bien, se basa en la experiencia del médico en tanto tal, la cual remite tanto a su conocimiento teórico como a la percepción (aỉo $\theta \dot{\sigma} \sigma \varsigma)$ del paciente, puesto que ambos son necesarios para el ejercicio de la medicina, $y$, en ese sentido, ni el planteamiento de la etiología ni la proposición del procedimiento por analogía en DM $\$ 22$ serían problemáticos para el empirismo del autor de este tratado, ya que, contrariamente a como sería en las prácticas (દ̉ $\mu \pi \varepsilon ı$ píaı) según Platón, su

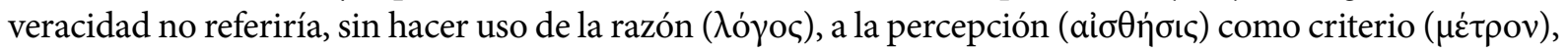

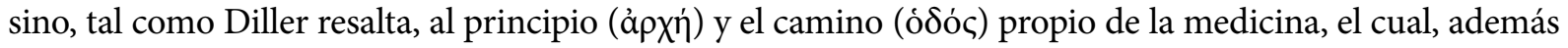
de lo puramente perceptual, dispondría de teorizaciones basadas en la percepción.

Finalmente, cabe señalar que sostener en DM un empirismo en el que la restricción empírica de la medicina no sea del modo anterior, i.e., la privación de teorización racional alguna, sino la imposibilidad de la teorización propia de la medicina, de modo que, al poder recurrirse en el ejercicio médico a

82 Sigo en la traducción de este término a Lara Nava.

83 DM $\$ 22.1-4$.

$84 \mathrm{DM} \$ 2.10-20$.

$85 \mathrm{DM} \$ 9.11-13$.

86 DM $\$ 12.10-13$.

87 Schiefsky, "Hipp. On An. Med., 345-359.

$88 \mathrm{DM} \$ 2.10-20$. 
teorizaciones ajenas a la medicina, no se estaría contradiciendo a DM $\$ 12$, tampoco sería posible, puesto que, asumiendo este planteamiento del empirismo, los planteamientos teóricos de la medicina, i. e, sus esquemas, tendrían que sostenerse sobre doctrinas ajenas a esta, y en otro orden de cosas, no podría plantearse la independencia de la medicina, tal como se hace en DM $\$ 1$ y 2 , secciones en las que, tal como señala Diller, para presentar la idea de la existencia genuina de la medicina como técnica ( $\tau \varepsilon ́ \chi v \eta)$, se hace hincapié en el hecho de que esta tiene un principio (ả $\left.\rho \chi \eta^{\prime}\right)$, tal como se lo entiende en $\mathrm{DC}^{89}, \mathrm{y}$

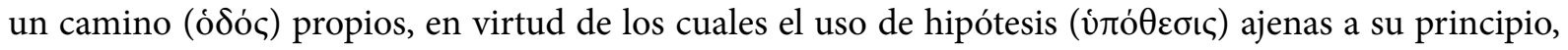
camino, método y esquemas es innecesario.

Dicho esto, el empirismo de DM sería uno que remite a la "experiencia técnica" del médico en tanto tal, la

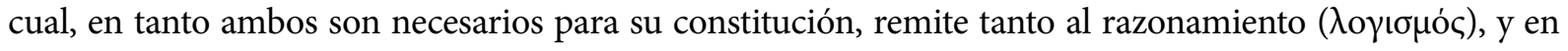
este sentido al conocimiento teórico médico, como a la percepción (aỉ $\theta \dot{\sigma} \sigma \varsigma$ ) del paciente, de manera que, en principio, ni la etiología ni el planteamiento de la analogía en el diagnóstico de enfermedades no visibles

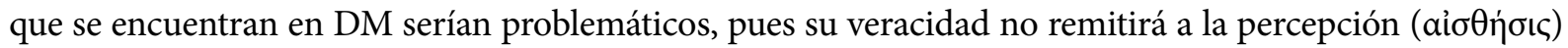
sin más, sino a la experiencia propiamente médica, tal como la he planteado, es decir, como una experiencia que en tanto propiamente médica no recurre en su ejercicio a planteamientos que le son ajenos e implica el

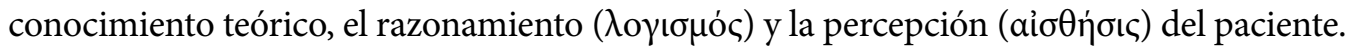

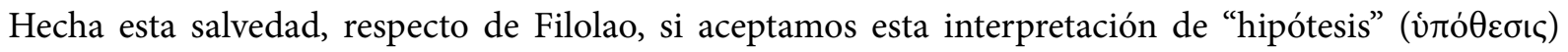
en DM, planteamos la crítica en este tratado de este modo y sostenemos, junto con Huffman, que de los principios (ả $\rho \chi \alpha i)$ deben ser entendidos en los fragmentos como "principios explicativos", $i$. e., condición lógica necesaria para que se dé el ámbito de los fenómenos, es decir, las cosas que pueden ser conocidas por el hombre, la crítica podría estar dirigida a Filolao, ya que, tal como se señaló en $\$$ II, este somete a comprobación su doctrina, de modo que supone la comprobabilidad de sus tesis, y el procedimiento por el cual concluye tales principios explicativos, en tanto condiciones lógicas necesarias, sería, en principio, ajeno a un método empírico. Así, lo mismo se podría comentar acerca de la doctrina médica que encontramos en DK 44 A 27, dado que, sobre la base de la analogía planteada por Huffman, esta se remitiría a la doctrina filosófica, que, según esta interpretación de Filolao, sería presentada en los fragmentos auténticos a partir de un método puramente lógico. Sin embargo, si se sostiene una interpretación de DK 44 B 2 en la que, basados en que en este fragmento se hace referencia a

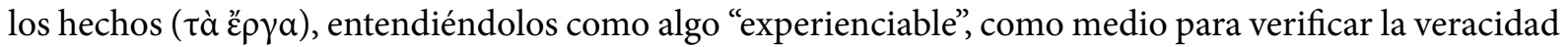
del planteamiento, de manera que en los fragmentos auténticos se estaría concluyendo su doctrina cosmología y cosmogónica no solo por medio de la argumentación, sino también por medio de un método empírico, la crítica formulada en DM no podría estar dirigida a Filolao como filósofo, en tanto este no plantearía su doctrina exclusivamente a partir de un modo ajeno al método empírico que, según lo dicho en DM, es pertinente también en los asuntos propios de la filosofía.

Ahora bien, a Filolao como médico la crítica de DM podría ser dirigida solo parcialmente, pues, por un lado, la crítica planteada en DM \$2. 4-7 le sería pertinente, puesto que, si aceptamos la analogía que propone Huffman entre lo caliente y lo frío en el testimonio DK 44 A 27 y los limitantes e ilimitados en DK 44 B 1,2 y 6, este en el planteamiento de lo caliente y lo frío de DK 44 A $27^{90}$ como causas de las

89 DC \$1. 1-8.

90 Como se resaltó en la introducción, aunque, de hecho, en DM $\$ 13-19$ se critica exclusivamente la suposición de lo frío y lo caliente como causas de las enfermedades, es necesario recordar que en esta parte de DM se critica el empleo 
enfermedades, análogamente al planteamiento de DF, ignoraría el aparato teórico, el "punto de partida" (ả $\rho \chi \eta ́)$, propio de la medicina, y la crítica en DM $\$ \$ 13-19$ demostraría que tales planteamientos son superfluos al ejercicio de la medicina, dado que no darían cuenta del origen de todas las enfermedades ni serían útiles en el diagnóstico y el tratamiento; por otro, según lo dicho, aun cuando, tal como se ha señalado, se podría plantear que estos son algo experienciable y, en ese sentido, se puede sostener que esta tesis se concluye a partir de un método empírico y uno hipotético. Solo, asumiendo que en DK 44 B 2 los hechos ( $\tau \grave{a}$ ép $\rho \alpha$ ) son entendidos como hechos lógicos, se podría atribuir el uso exclusivo del método hipotético, el cual sería objeto de crítica en DM, a Filolao en el planteamiento de la tesis de la existencia de los principios (ảpxaí) que estaría en juego en su doctrina filosófica y médica.

En consecuencia, por un lado, si se sostiene una interpretación "lógica" de los fragmentos DK 44 B 2 y 6 , la analogía planteada por Huffman entre los limitantes e ilimitados en DK 44 B 1, 2 y 6, y lo caliente y lo frío en DK 44 A 27, y se plantea la crítica en DM, entendiendo "hipótesis" (ن́ró $\theta \varepsilon \sigma ı)$ ) como proposición que debe ser comprobada, como una crítica metodológica al método hipotético, puesto que, al tener un "punto de referencia" o un criterio para la verificación de la veracidad de las proposiciones, solo el método empírico es un medio apropiado para obtener conocimiento, esta podría ser dirigida a Filolao en tanto filósofo y como médico, pues este plantearía los principios que estarían en juego tanto en su doctrina filosófica como en la médica, en virtud de la necesidad lógica; por el otro, siguiendo una interpretación de DK 44 B2 que no restringe el planteamiento de Filolao al ámbito puramente lógico, a pesar de que las críticas planteadas en DM $\$ 2$. 8-9 y en $\$ 20$. 8-9 no podrían ser dirigidas a Filolao ni como filósofo ni como médico, pues este no plantearía su doctrina ni filosófica ni médica de manera ajena al método empírico, las críticas que se plantean en DM $₫ 13-19$ y $\$ 2$. 4-7 sí podrían ser dirigidas a Filolao en

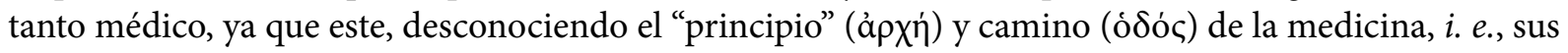
esquemas y planteamientos teóricos, plantearía según su teoría filosófica una doctrina médica que, tal como se concluye en DM $\$ 13-19$, sería superflua en la práctica médica.

\section{IV}

La última posible interpretación del término "hipótesis" (نં

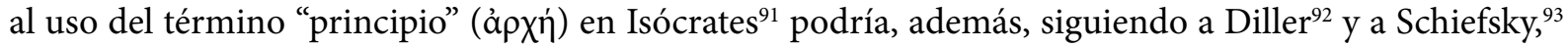
ser planteada según un número significativo de pasajes de autores del siglo IV a. C., en que "principio"

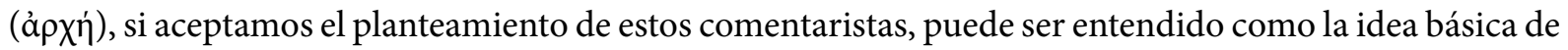
un discurso, i. e., su objeto o su tesis, o como un principio fundamental que conduce una cierta actividad, es decir, su finalidad. Ahora bien, si aceptamos esta interpretación del término, podríamos sostener que

de hipótesis (úđó $\theta \varepsilon \sigma \iota \varsigma)$, independiente de la interpretación que se le dé a este término, en la medicina y que, en ese sentido, la crítica no es meramente a postulación de lo frío y lo caliente como causas de las enfermedades, sino a esta postulación como ejemplo de los planteamientos que sostienen aquellos que hacen uso de tales hipótesis (ن́ró $\theta \varepsilon \sigma \iota \varsigma)$ en la medicina. Así, empero, junto con Huffman, Lloyd y Schiefsky, que se esté refutando en estas secciones del tratado una doctrina según la cual lo frío y lo caliente son causas de las enfermedades no es evidencia suficiente para deducir que es Filolao el oponente que tendría en mente el autor de DM.

91 Pan. 63.

92 Diller, “Hipp. Med.Att. Phil.", 387-388.

93 Schiefsky, “Hipp. On An. Med., 111-112. 


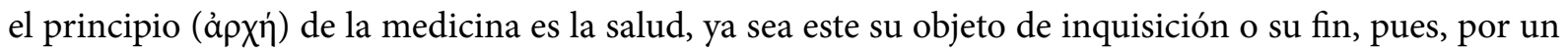
lado, tal como se señala en DM $\$ 2$, lo que concierne a la investigación médica son los padecimientos de los pacientes, sus causas y curas; $;^{94}$ por otro, según DM $\$ 3$, el descubrimiento de la medicina se hizo con miras a la salud. ${ }^{95}$ Así pues, la crítica en DM podría ser leída como una que está dirigida a un

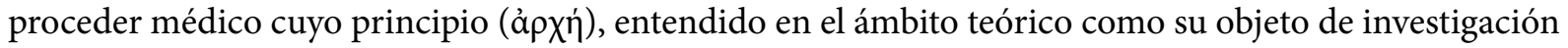
y en el práctico como su finalidad, no es la salud. Si esto es así, cabe señalar que solo el aspecto teórico del término principio (ả $\rho \chi \eta ́)$ estaría en juego en De prisca medicina, puesto que en DM no se critica a médicos que no busquen mediante el diagnóstico y tratamiento de los pacientes restablecer o preservar su salud, sino a aquellos que, tal como nos dice el autor en $\mathrm{DM} \$ 2$, ignorando en su práctica los métodos y esquemas propios de la medicina, basan su doctrina médica en tesis que son ajenas a esta; ${ }^{96}$ usando los términos de Schiefsky ${ }^{97}$, fundamentan su ejercicio médico según una embriología o antropogonía. En consecuencia, la crítica del autor de DM a sus oponentes no es que estos no tengan como finalidad la salud, sino que, con este fin en mente, tal y como se dice en DM $\$ 20$, supongan como necesaria la inquisición sobre asuntos que no conciernen a la salud, i. e., sobre el origen y constitución del hombre ${ }^{98}$, $y$, en ese sentido, tal como señala Schiefsky ${ }^{99}$, la crítica en DM no supondría una crítica a la filosofía, sino al influjo filosófico en la medicina.

Dicho esto, los oponentes de DM sostendrían una concepción de la medicina como la que encontramos en DC, pues, dado que solo por medio de tales inquisiciones se llegaría a conocer cómo se originaron y llegaron a ser el hombre y otras cosas vivientes, qué es el alma, qué es la salud y la enfermedad, qué es lo bueno y lo malo para el hombre y la razón de su muerte, se plantea una concepción de la medicina en la

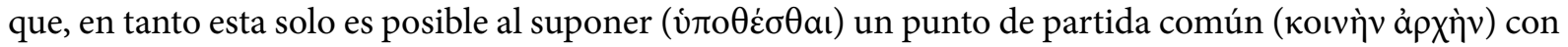
aquellos que investigan acerca de los asuntos del cielo, ${ }^{100}$ se asevera, contrariamente a lo que se nos dice en DM, que es necesaria para la técnica ( $\tau \dot{\varepsilon} \chi \vee \eta)$ médica la inquisición que, en principio, es ajena a esta sobre asuntos tales como el origen de los seres vivientes o el alma, i. e., desde el punto de vista de DM, los asuntos del cielo y de debajo de la tierra. ${ }^{101}$

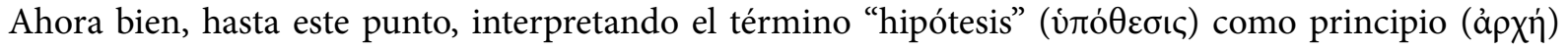
"teórico", i. e. como objeto de inquisición, se ha planteado la crítica en DM como una crítica exclusivamente médica que no estaría dirigida a la filosofía o a los planteamientos filosóficos en tanto tales. Sin embargo, a esta formulación de la crítica en DM se puede objetar que en DM $\$ 2$ y 20, en tanto se asevera que solo por medio de la medicina es posible el conocimiento, incluso, de aquello que, en esta interpretación de

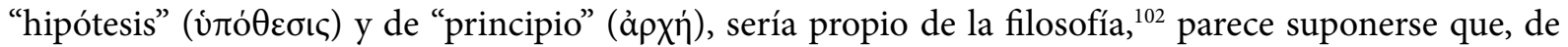
hecho, el objeto de inquisición de la medicina y la filosofía es el mismo, de modo que, aunque en DM $\$$

$94 \mathrm{DM} \$ 2.10-20$.

$95 \mathrm{DM} \$ 3.36-40$.

$96 \mathrm{DM} \$ 2.4-7$.

97 Schiefsky, "Hipp. On An. Med., 19-25; Schiefsky, “On Anc. Med. on the nat. of human beings", 69-85.

98 DM $\$ 20.1-8$.

99 Schiefsky, "Hipp. On An. Med., 55-62.

$100 \mathrm{DC} \$ 1.1-8$

$101 \mathrm{DM} \$ 1.15-21$.

$102 \mathrm{DM} \$ 2.8-9$ y 20. 8-9. 
2 se sostiene que lo pertinente a la medicina son los padecimientos de los pacientes ${ }^{103}$, el conocimiento médico no tendría que limitarse a lo referente a la salud o tendría que sostenerse que el ámbito de la investigación filosófica, al menos en último término, remite a la inquisición sobre lo que es pertinente a la medicina que, siguiendo lo dicho en DM $\$ 20$, tiene que ver con qué es el hombre, no tomado en sí mismo, sino en relación con todo aquello con lo que interactúa. ${ }^{104}$ Así pues, la versión "teórica" de la crítica no se podría sostener tal como se la ha planteado hasta ahora, puesto que, si se entiende principio

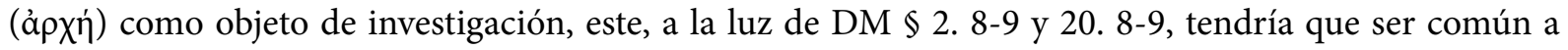
ambas, de modo que la crítica a la influencia filosófica de los médicos que son oponentes del autor de DM no podría formularse como una "intromisión" en el campo de lo pertinente a la medicina, sino que más bien tendría que formularse como una crítica a los filósofos, que, ignorando que la filosofía es, de hecho, una cierta medicina, se embarcan en investigaciones ajenas a esta, las cuales, según lo dicho en DM $\$ 20$, tendrían que ver más bien con la literatura ( $ү \rho а \varphi \iota \kappa \eta ́) .105-106$

Además, cabe señalar que también sería posible plantear la crítica en DM entendiendo principio (ảpxí) como finalidad, pues esta ya no estaría dirigida a médicos que no practican la medicina en vistas a la salud, sino a filósofos que, nuevamente, ignorando que la filosofía comparte finalidad con la medicina no filosofan con el objetivo de restaurar o mantener la salud. ${ }^{107}$ No obstante, es necesario recalcar que en ningún momento en DM se plantea una crítica a la filosofía desde el punto de vista de tender a algo que es ajeno a su finalidad, sino que la argumentación de DM contra la filosofía buscaría, siguiendo esta interpretación de "hipótesis" ( objeto de inquisición, demostrar que la medicina es independiente de la filosofía, ${ }^{108}$ y si consideramos lo dicho en DM $\$ 2.8-9$ y 20. 8.9, al mostrar que las doctrinas planteadas en las investigaciones sobre asuntos ajenos a lo respecta al hombre en relación con las cosas con las que interactúa son inútiles tanto en la medicina como en la filosofía y que solo a partir de la inquisición médica sería posible conocer

$103 \mathrm{DM} \$ 2.10-20$.

104 Una inquisición de este tipo le atribuye Kahn en su interpretación a Heráclito. Véase Kahn, “The Thesis of Parmenides”, 9-23.

105Sigo en la traducción de este término a Lara Nava quien llama la atención en que este también puede referirse a la pintura, de modo que podría relacionarse la idea de que la filosofía tiene que ver con la pintura que encontraríamos en esta frase de DM $\$ 20$ con la comparación entre la formación de los seres a partir de los elementos con el trabajo del pintor que plantearía Empédocles, a quien se hace alusión explícita en DM, en el fragmento DK 31 B 23. Por su parte, La Croce ratifica el planteamiento de esta comparación en este fragmento de Empédocles y señala que, a la luz de DK $31 \mathrm{~A}$ 93, podría plantearse la analogía no solo en el proceso de formación de los seres, sino también respecto de los elementos mismos, pues, según este testimonio, Empédocles equipararía los elementos con los colores básicos que, siguiendo lo dicho en De mundo por Ps. Aristóteles, se usarían en la pintura ( $(\omega \gamma \rho \alpha \varphi i ́ a)$, a saber, el blanco, el negro, el rojo y el amarillo. Véase Lara Nava, “Sobre la medicina antigua”, 161, n. ${ }^{\circ}$ 40; La Croce, “Empédocles de Agrigento”, 186, n. 97.

$106 \mathrm{DM} \$ 20.8$.

107Concepciones de la filosofía como cierto tipo de medicina las encontramos en la filosofía helenística, e. g., en la Carta a Meneceo, Epicuro nos presenta como el fin o, siguiendo la interpretación que se ha dado al término $\$$ IV., como el

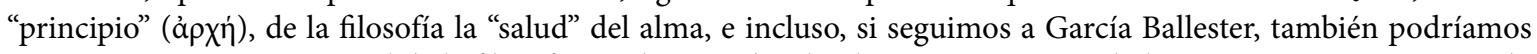
encontrar una concepción tal de la filosofía en el Cármides, de Platón, pues en este diálogo se estaría caracterizando la virtud, i. e., el fin de la filosofía moral, como salud del alma y las opiniones erróneas como enfermedad. Además, se podría agregar a este planteamiento que en este mismo diálogo se presenta a Sócrates, i. e., un filósofo, sino como un médico, como alguien quien procede del modo en que un buen médico lo haría, es decir, alguien que actuaría con miras a la salud. Véase Cart. Men. 122 1-3, Carm. 155c7-157c 6 y García Ballester, "Soul and Body”, 117-152.

$108 \mathrm{DM} \$ 3-7$. 
algo acerca de la naturaleza, concluir que la filosofía depende de la medicina. En otro orden de cosas, aunque una crítica a la filosofía en virtud de su finalidad es posible sobre esta interpretación del término

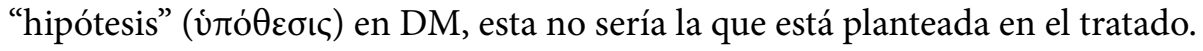

Llegados a este punto, podría leerse la crítica en DM a la luz del planteamiento del objeto de la medicina planteado en DM $\$ 20$ como una dirigida tanto a filósofos como a médicos que investigan acerca de la naturaleza planteando como principios (ả $\rho x \eta ́)$ teóricos los elementos o las nociones matemáticas y

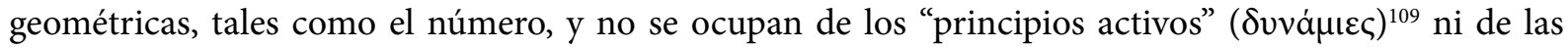
relaciones entre ellos, de modo que, según DM $\$ 1$, no tendrían criterio para verificar la veracidad de sus doctrinas. ${ }^{110}$ Dicho esto, la crítica podría estar dirigida a médicos tales como el autor de DF, pues, en este tratado, se concluye que el aire, es decir, un elemento, es causa de todas las enfermedades; el de DC, quien plantea que la medicina solo es posible en referencia a doctrinas filosóficas que remiten a los elementos; y en otro orden de cosas, al ocuparse de la medicina plantea, al menos en principio, una cosmogonía y antropogonía elemental en la que da cuenta detalladamente de cómo se formaron y de qué se componen los órganos, ${ }^{111} \mathrm{o}$ uno que suponga el estudio del número o de las relaciones matemáticas o geométricas como base para el conocimiento médico o filosófico. ${ }^{112}$

Ahora bien, en lo que respecta a Filolao, no es del todo claro que este, entendiendo el término "hipótesis" (v́ró $\theta \varepsilon \sigma ı \varsigma)$ como objeto de investigación, pueda ser el que es atacado en la argumentación de DM, pues, si seguimos una interpretación "elemental", como la que se ha planteado hasta ahora y que sostienen Huffman, ${ }^{113}$ Lloyd ${ }^{114}$ Schiefsky ${ }^{115}$ y Sassi, ${ }^{116}$ la crítica, así planteada, podría estar dirigida a Filolao como

109La noción de elementos que es ejemplificada en este tratado como "lo caliente y lo frío" o "lo seco y lo húmedo", y la

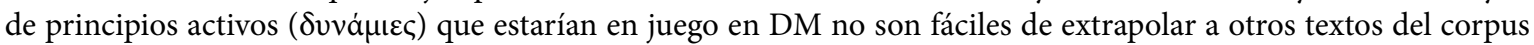
hipocrático, pues en varios tratados hipocráticos tales como DF, DC, De natura hominis $(\mathrm{NH})$ o De aere aquis et locis (AAL) se atribuye a los elementos tales como lo caliente, lo frío, lo seco y lo húmedo la característica fundamental que es atribuida a los principios activos en DM $\$ 20$, a saber, afectar al hombre de maneras determinadas. Lo mismo sucede en autores que son reconocidos como filósofos como Alcmeón, quien en el fragmento DK 24 B 4 denomina indiscriminadamente tanto a lo seco, lo húmedo, lo caliente, lo frío, es decir, lo que en DM sería, si aceptamos la interpretación que se ha plateado del tratado, presentado como los elementos, como a lo dulce y lo agrio, $i$. e., lo

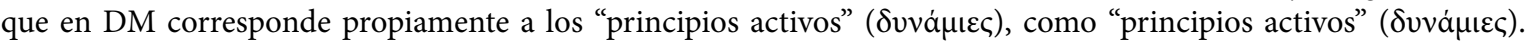
Sin embargo, tal como hace Mansfeld, es necesario señalar que estos términos podrían poner en tela de juicio la autenticidad del fragmento DK 24 B 4, en tanto serían "terminología doxográfica” que no sería propia de Alcmeón y no podría ser atribuida a él. Véase DM $₫ 20.17-23, \S 14$ 20-27; y Mansfeld, “The Body Politic”, 78-95.

$110 \mathrm{DM} \S 1.15-21$.

$111 \mathrm{DC} \S 2-14$.

112Esta caracterización de los oponentes de DM puede concordar, si seguimos a Huffman, con algunas presentaciones que hace Aristóteles de los pitagóricos, a quienes, según el planteamiento del comentarista, este les atribuiría, al menos en Met. 1083b 16-19 y en Met. 985b 23-986a 6, además, la doctrina de que los principios (ả $\rho x a i)$ de los objetos matemáticos $(\mu \alpha \theta \dot{\mu} \mu \alpha \tau \alpha)$ son principio de todas las cosas o bien todas las cosas con número, la práctica de aplicar

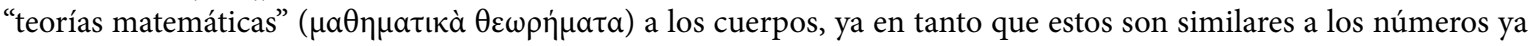
porque son números. Véase Huffman, “The Role of Number in Philolaus' Philosophy”, 8-11.

113 Huffman, "Philolaus of Croton: Pythagorean and Presocratic", 37-53; Huffman, “The Role of Number in Philolaus' Philosophy", 19-30.

114Lloyd, "Who Is Attacked in 'On Ancient Medicine'?”, 118-126.

115Schiefsky, “Hipp. On An. Med., 52-62.

116Sassi, “How Musical was Heraclitus’ Harmony?”, 10-16. 
filósofo y como médico, ya que, aun sin sostener la analogía que propone Huffman ${ }^{117}$ entre lo caliente y lo frío en DK 44 A 27 y los limitantes e ilimitados en DK 44 B 1,2 y 6, en cada una de estas áreas se plantearían doctrinas "elementales". No obstante, si no se sostiene una interpretación "elemental", sino una como la que propone Fotini Viltanioti ${ }^{118}$ en la que los limitantes y los ilimitados se entienden como "poderes", i. e., "principios activos", y no como "elementos", la crítica, en principio, no podría estar dirigida a Filolao, puesto que este en los fragmentos auténticos, y si aceptamos el planteamiento de Huffman, en DK 44 A 27 no se ocuparía de elementos, sino, como ya hemos dicho, de los "principios activos". Por otra parte, si, siguiendo esta interpretación de Filolao, no se acepta la propuesta de Huffman, se podría llegar a plantear, como hace Lloyd, que el Filolao de DK 44 A 27, en tanto la basaría sobre la idea de que lo caliente y lo frío son los principios últimos (ả $\chi \alpha i$ ) de las enfermedades, plantearía su teoría médica remitiendo doctrinas filosóficas elementales y, en ese sentido, podría ser el oponente de la crítica en DM, tal como la hemos planteado.

Finalmente, cabe resaltar que, si consideramos que en DK 44 B 4 y DK 44 A 7a, en que, tal como se ha señalado en $§ I I$, si seguimos a Huffman, ${ }^{119}$ se estaría sosteniendo que el número es principio (ảpx门́) de

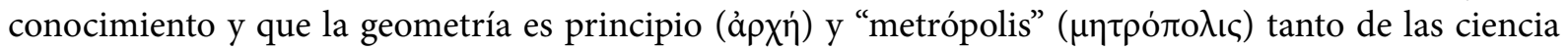
matemáticas como de la filosofía y la medicina, ${ }^{120}$ se podría afirmar, junto con Lloyd, que Filolao es el oponente del autor de DM, en tanto este, ya sea que se sostenga en los fragmentos auténticos una interpretación "elemental" de los principios (ả $\rho \chi \alpha^{\prime}$ ), o una en la que estos se deben entender como "principios activos" ( $\delta v v a ́ \mu t \varepsilon \varsigma)$, a la luz del fragmento y el testimonio ya citados, supondría como base "teórica" para la inquisición médica a la geometría, es decir, partiría en la formulación de sus teorías tanto médicas como filosóficas de una investigación, cuyo objeto de estudio son las relaciones matemáticas entre los objetos y del número. ${ }^{121}$

\section{V}

En conclusión, habiendo asumido cada una de las posibles interpretaciones del término "hipótesis" (vंró $\theta \varepsilon \sigma \iota \varsigma)$ en DM que menciona Loyd, se ha demostrado, por una parte, que, dado que en los fragmentos

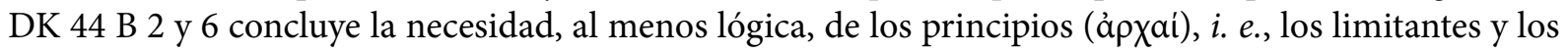
ilimitados, y de la armonía (áphovía), Filolao no supondría la incomprobabilidad de estas proposiciones y que tampoco mantiene sus conclusiones como meras propuestas que no llega a afirmar, si se acepta

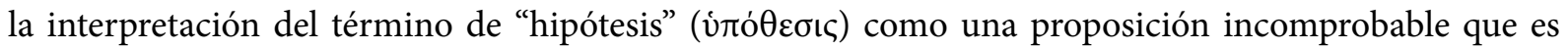
propuesta, pero que no puede ser afirmada y como tal superflua en la investigación médica o filosófica, Filolao no podría ser el oponente de DM, pues las conclusiones que este sostiene no supondrían el carácter superfluo que sería atribuido en DM a las hipótesis.

117 Huffman, "Philolaus of Croton: Pythagorean and Presocratic", 41-47.

118Fotini Viltanioti, “Powers as the Fundamental Entities in Philolaus' Ontology”, 10-29.

119Huffman, "Philolaus of Croton: Pythagorean and Presocratic", 193-199; Huffman, "The Role of Number in Philolaus' Philosophy", 19-30.

120 Véase la nota al pie de página 63.

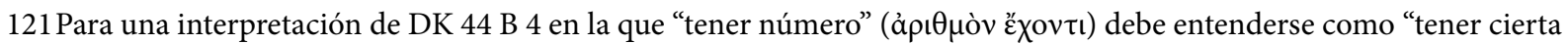
estructura o ciertas relaciones matematizables", véase Huffman, "Philolaus of Croton: Pythagorean and Presocratic" y “The Role of Number in Philolaus' Philosophy”. 
Por esta misma razón, a saber: que en los fragmentos DK 44 B 2 y 6 demuestra la necesidad, al menos lógica, de los principios (ả $\rho \chi a i)$, i. e., los limitantes y los ilimitados, y de la armonía (á $\rho \mu o v i ́ a)$, se ha concluido que, si planteamos la crítica en DM entendiendo "hipótesis" (író $\theta \varepsilon \sigma \iota \varsigma)$ como un supuesto, i. e., como una proposición cuya veracidad es supuesta, esta no podría estar dirigida a Filolao como filósofo, en tanto que no plantearía su doctrina siguiendo el método hipotético. Por el contrario, se ha concluido que a Filolao como médico sí podría estar dirigida esta crítica, puesto que este, aun si no seguimos la analogía planteada por Huffman ${ }^{122}$ entre los limitantes e ilimitados en DK 44 B 1, 2 y 6, y lo caliente y lo frío en DK 44 A 27, estaría, en modo análogo al autor de DC, formulando su teoría

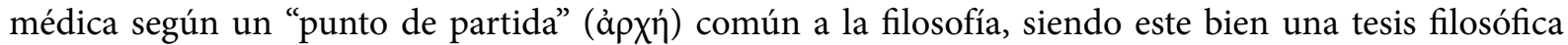
como lo sería la necesidad de armonía (à $\mu$ ovía), o bien, como sugieren Lloyd y Huffman en referencia

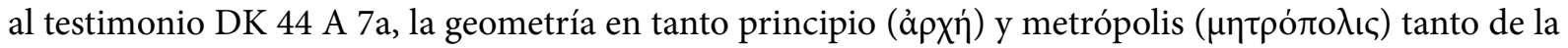
filosofía como de la medicina, y a la luz de DK 44 B 4 y como sugiere con Huffman, el número como principio epistemológico.

Por otra parte, se ha demostrado que, si se asume, junto con Huffman, una interpretación puramente lógica

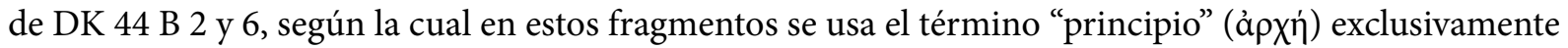
como principio explicativo o condición lógica necesaria, y se entiende "hipótesis" (ن́đó $\theta \varepsilon \sigma \iota \varsigma)$ en DM como proposición que debe ser comprobada, de modo que la crítica en este tratado estaría formulada como una crítica metodológica al método hipotético, en tanto, al tener un "punto de referencia" o un criterio para la verificación de la veracidad de las proposiciones, solo el método empírico sería un medio apropiado para obtener conocimiento, esta podría ser dirigida a Filolao en tanto filósofo y como médico, pues este plantearía los principios que estarían en juego tanto en su doctrina filosófica como en la médica en virtud de la necesidad lógica. No obstante, si se sigue una interpretación de DK 44 B 2 que no restringe el planteamiento de Filolao al ámbito puramente lógico, aunque las críticas que se plantean en DM $\$ 13-19$ y $\$ 2$. 4-7 sí podrían ser dirigidas a Filolao en tanto médico, ya que este plantearía su

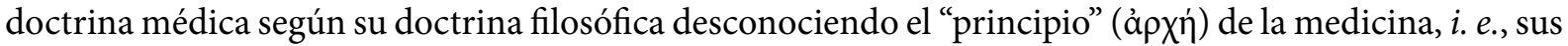
esquemas y planteamientos teóricos, y tanto sus doctrinas como los principios filosóficos de los que parte su planteamiento médico serían superfluos en la práctica médica, la crítica metodológica no podría ser dirigida a Filolao como filósofo ni como médico, dado que este no plantearía su doctrina ni filosófica ni médica de manera ajena al método empírico.

Finalmente, se ha concluido que, si se sigue una interpretación "elemental" de Filolao, como la que

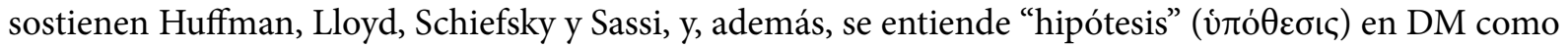
principio teórico, i.e., como objeto de inquisición, la crítica en este tratado podría estar dirigida a Filolao como filósofo y como médico, puesto que, aun si no se sostiene la analogía que propone Huffman entre lo caliente y lo frío en DK 44 A 27 y los limitantes e ilimitados en DK 44 B 1,2 y 6, en cada una de estas áreas este plantearía doctrinas "elementales", y que, si no se sostiene una interpretación "elemental", sino una como la que propone Fotini Viltanioti en la que los limitantes y los ilimitados se entienden como "poderes", i. e., "principios activos", esta crítica no podría estar dirigida a Filolao ni como filósofo ni, si aceptamos la analogía que plantea de Huffman, como médico, ya que tanto en los fragmentos

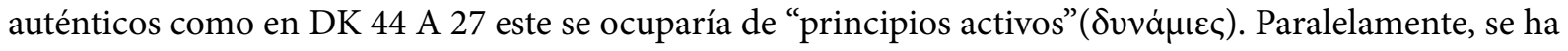
demostrado, por un lado, que, si, siguiendo esta interpretación de Filolao, no se acepta la propuesta de Huffman, se podría llegar a plantear, como hace, que Filolao en DK 44 A 27 basa su teoría médica sobre

122Huffman, "Philolaus of Croton: Pythagorean and Presocratic", 41-47. 
lo caliente y lo frío, i. e., en elementos, de modo que este podría ser el oponente a quien está dirigida la crítica en DM formulada sobre la interpretación de "hipótesis" (v́đó $\theta \varepsilon \sigma ı)$ ) como principio teórico, pues esta estaría dirigida a aquellos filósofos o médicos que se ocupan de los elementos y no de los principios activos ( $\delta v v a ́ \mu t \varepsilon \varsigma)$, los cuales serían, siguiendo esta interpretación, el objeto de inquisición de la filosofía y de la medicina; por otro, que, entendiendo en DM el término "hipótesis" (v́đó $\theta \varepsilon \sigma \iota \varsigma)$ como principio

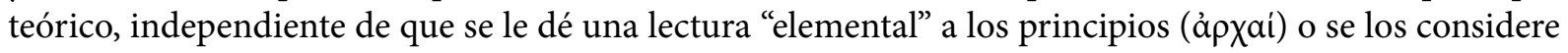

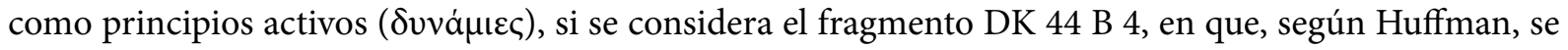
sostiene que el número es principio del conocimiento, y el testimonio DK $44 \mathrm{~A} 7 \mathrm{a}$, en que, de acuerdo con Huffman, se plantea la primacía de la geometría respecto de cualquier investigación, podría afirmarse junto con Lloyd que Filolao es el oponente del autor de DM, en tanto este partiría en el planteamiento de sus doctrinas filosóficas médicas de la inquisición sobre un objeto ajeno a la investigación médica, i.e., las relaciones matemáticas entre los objetos.

\section{Bibliografía}

\section{Ediciones y traducciones}

Bekker, Immanuel, ed. Aristóteles: Graece ex recensione Immanuelis Bekkeri. Vol. 1. Berlín: Georg Reimer, 1831. Bekker, Immanuel, ed. Aristóteles: Graece ex recensione Immanuelis Bekkeri. Vol. 2. Berlín: Georg Reimer, 1831. Burnet, John, ed. Platonis opera. Vol. 2. Nueva York: Oxford University Press, 1900.

Burnet, John, ed. Platonis opera. Vol. 3. Nueva York: Oxford University Press, 1900.

Burnet, John, ed. Platonis opera. Vol. 4. Nueva York: Oxford University Press, 1900.

Calonge Ruiz, Julio, ed. Gorgias en Platón. Diálogos. Vol. 2. Editado por Carlos García Gual y José Luis Navarro, 8-145. Madrid: Gredos, 1992.

Calvo Martínez, Tomás, ed. Aristóteles. Metafísica. Madrid: Gredos, 1994.

Candel, Miguel, ed. Acerca del cielo en Aristóteles. Acerca del cielo/Meteorológicos. Editado por Daniel Riaño Rufilanchas, 9-228. Madrid: Gredos, 1996.

Cano Cuenca, Jorge, ed. Sobre la naturaleza del hombre en Tratados hipocráticos. Vol. 8. Editado por Oscar Martínez García y Mario Toledano, 11-64. Madrid: Gredos, 2003.

De la Villa Polo, Jesús, ed. Sobre las carnes en Tratados hipocráticos. Vol. 8. Editado por Oscar Martínez García y Mario Toledano, 137-164. Madrid: Gredos, 2003.

Diels, Hermann, ed. Anonymus Londinensis: Auszüge eines Unbekanntes aus Aristóteles-Menons Handbuch der Medizin und aus Werken anderer älterer Aerzte. Berlín: Georg Reimer, 1896.

Diels, Hermann y Walther Kranz, ed. Die Fragmente der Vorsokratiker. Griechisch und Deutsch. Vol. 3. Berlín: Weidmannsche Verlagsbuchhandlung, 1960.

Duke, E. A., W. F. Hicken, W. S. M. Nicoll, D. B. Robinson y J. C. G. Strachan, eds. Platonis opera. Vol. 1. Nueva York: Oxford University Press, 1995.

Durán, María Ángeles, ed. Filebo en Platón. Diálogos. Vol. 6. Editado por Carlos García Gual y Mercedes López Salvá, 7-124. Madrid: Gredos, 1997.

Eggers Lan, Conrado, ed. Filolao y los llamados pitagóricos en Los filósofos presocráticos. Vol. 3. Editado por Alberto del Pozo Ortiz, 77-140. Madrid: Gredos, 1986.

Eggers Lan, Conrado, ed. República en Platón. Diálogos. Vol. 4. Editado por Alberto del Pozo Ortiz, 57-497. Madrid: Gredos, 1986.

Walzer, Richard y Michael Frede, eds. Galen: Three treatises On the Nature of Science. Indianápolis: Hackett Publishing Company, 1985.

García Gual, Carlos, ed. Sobre la ciencia médica en Tratados hipocráticos. Vol. 1. Editado por Carlos García Gual y Elsa García Novo, 99-122. Madrid: Gredos, 1983. 
García Gual, Carlos, ed. Fedón en Platón. Diálogos. Vol. 3. Editado por Luis Alberto de Cuenca y Prado, Carlos García Gual y José Luis Navarro, 7-142. Madrid: Gredos, 1988.

García Gual, Carlos, ed. Parménides en Platón. Diálogos. Vol. 5. Editado por Carlos García Gual y Fernando García Romero, 7-136. Madrid: Gredos, 1988.

Gottlob Kühn, Karl, ed. ГA $\Lambda$ HNOY ПEPI AIPE $\Sigma$ E $\Omega$ N TOI $\Sigma$ EI $\Sigma$ AГOMENOI $\Sigma$. Galeni de sectis ad eos qui

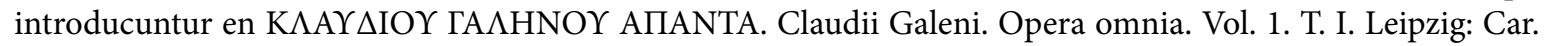
Cnoblochii, 1821.

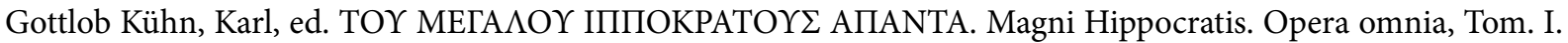
T. I. Leipzig: Car. Cnoblochii. 1825.

Huffman, Carl A., ed. Philolaus of Croton. Pythagorean and Presocratic: A Commentary on the Fragments and Testimonia with Interpretive Essays. Nueva York: Cambridge University Press, 1993.

Huffman, Carl A., ed. Archytas of Tarentum: Pythagorean, Philosopher and Mathematician King. Nueva York: Cambridge University Press, 2005.

Kahn, Charles H., ed. The art and thought of Heraclitus: An edition of the fragments with translation and commentary. Nueva York: Cambridge University Press, 2004.

La Croce, Ernesto, ed. Empédocles de Agrigento en Los filósofos presocráticos. Vol. 2. Editado por Alberto del Pozo Ortiz, 129-296. Madrid: Gredos, 1985.

Lara Nava, María Dolores, ed. Sobre la medicina antigua en Tratados hipocráticos. Vol. 1. Editado por Carlos García Gual y Elsa García Novo, 123-168. Madrid: Gredos, 1983.

López Férez, Juan Antonio, ed. Sobre los flatos en Tratados hipocráticos. Vol. 2. Editado por María Dolores Lara Nava, 119-148. Madrid: Gredos, 1986.

López Férez, Juan Antonio, ed. Sobre los aires, las aguas y los lugares en Tratados hipocráticos. Vol. 2. Editado por María Dolores Lara Nava, 7-88. Madrid: Gredos, 1986.

Lledó Î́nigo, Emilio, ed. Cármides en Platón. Diálogos. Vol. 1. Editado por Pedro Bádenas y Carlos García Gual, 317-368. Madrid: Gredos, 1985.

Manzano Martínez, Teresa, ed. Sobre las escuelas de medicina, a los principiantes en Galeno. Tratados filosóficos y autobiográficos. Editado por David Hernández de la Fuente, 93-140. Madrid: Gredos, 2002.

Nornlin, George, ed. Isócrates. Vol. 1. Nueva York: William Heinemann, 1928.

Olivieri, Francisco J., ed. Menón en Platón. Diálogos. Vol. 2. Editado por Carlos García Gual y José Luis Navarro, 147-190. Madrid: Gredos, 1992.

Schiefsky, Mark J., ed. Hippocrates On ancient medicine. Traducido con introducción y comentario en Studies in ancient medicine. Vol. 28. Editado por Ann Hannson, John Scarborough, Nancy Siraisi y Philip J. van der Eijk. Boston: Brill, 2005.

Vara, José, ed. Epicuro. Obras completas. Madrid: Cátedra, 2012.

\section{Literatura secundaria}

Burnyeat, Myles. Plato on why mathematics is good for the soul en Mathematics and Necessity: Essays in the History of Philosophy. Proceedings of the British Academy. Vol. 103. Editado por Thomas Smiley, 1-81. Nueva York: Oxford University Press, 2000.

Diller, Hans. "Hippokratische Medizin und Attische Philosophie". Hermes 80, n. 4 (1952): 385-409.

Dunn, Francis. On ancient medicine and its intellectual context en Hippocrates in context. Papers read at the XIth International Colloquium University of Newcastle upon Tyne 27-31 August 2002. Editado por Philip J. van der Eijk, 49-68. Países Bajos: Brill, 2005. https://doi.org/10.1163/9789004377271_004

Fotini Viltanioti, Irini. "Powers as the Fundamental Entities in Philolaus' Ontology". Journal of Ancient Philosophy 6, no. 2 (2012): 1-30. https://doi.org/10.11606/issn.1981-9471.v6i2p1-31.

García Ballester, Luis. Soul and Body, Disease of the Soul and Disease of the Body in Galen's Medical Thought. Editado por Paola Manuli y Mario Vegetti, 117-152. Nápoles: Bibliopolis, 1988.

Huffman, Carl. "The Role of Number in Philolaus' Philosophy". Phronesis 33, no. 1 (1988): 1-30. https://doi. org/10.1163/156852888X00018

Kahn, Charles H. The Thesis of Parmenides en Essays on Being. Editado por Charles H. Kahn, 143-166. Nueva York: Oxford University Press, 2004. 
Lloyd, Geoffrey E. R. “Who Is Attacked in 'On Ancient Medicine'?”. Phronesis 8, no. 2 (1963): 108-126. https://doi. org/10.1163/156852863X00079

Longrigg, James, ed. Greek Medicine: From the Heroic to the Hellenistic Age. A Source Book. Nueva York: Routledge, 1998.

Mansfeld, Jaap. The Body Politic: Aëtius on Alcmaeon on Isonomia and Monarchia. Editado por Verity Harte y Melissa Lane, 78-95. Nueva York: Cambridge University Press, 2013. https://doi.org/10.1017/CBO9781139096843.006

Mourelatos, Alexander P. D. Parmenides: Revised and Expanded Edition. Míchigan: Parmenides Publishing, 2008.

Robinson, Richard. Plato's Earlier Dialectic. Cornell: Cornell University Press, 1941.

Sassi, Maria Michela. "How musical was Heraclitus' Harmony? A reassessment of 22 B 8, 10, 51 DK”. Rhizomata 1, n. 3 (2015): 3-25. https://doi.org/10.1515/rhiz-2015-0002.

Schiefsky, Mark J. On Ancient Medicine on the nature of human beings en Hippocrates in context. Papers read at the XIth International Colloquium University of Newcastle upon Tyne 27-31 August 2002. Editado por Philip J. van der Eijk, 69-87. Países Bajos: Brill, 2005. https://doi.org/10.1163/9789004377271_005

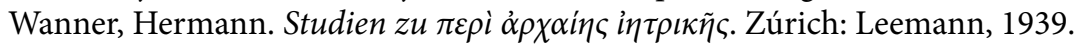

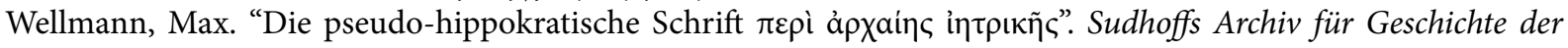
Medizin 23, no. 4 (1930): 299-305. 